\title{
Towards Personalized Models of the Cardiovascular System Using 4D Flow MRI
}

\author{
Belén Casas
}

Division of Cardiovascular Medicine

Department of Medical and Health Sciences

Linköping University, Sweden

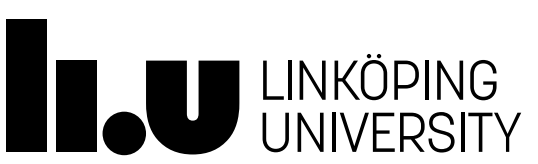




\section{Towards Personalized Models of the Cardiovascular System Using 4D Flow MRI}

Linköping University Medical Dissertations

Dissertation No.1641

Department of Medical and Health Sciences

Linköping University

SE-581 83, Linköping, Sweden

http://www.liu.se/cmr

Printed by:

LiU-Tryck, Linköping, Sweden

ISBN 978-91-7685-217-0

ISSN 0345-0082

Copyright () 2018 Belén Casas, unless otherwise noted

No part of this publication may be reproduced, stored in a retrieval system, or be transmitted, in any form or by any means, electronic, mechanic, photocopying, recording, or otherwise, without prior permission of the author.

Cover: Printed circuit board illustration of a human heart. 


\author{
Dices que tienes corazón, y sólo \\ lo dices porque sientes sus latidos. \\ Eso no es corazón...; es una máquina \\ que, al compás que se mueve, hace ruido. \\ Rima LXXVII, Gustavo Adolfo Bécquer
}





\section{Abstract}

Current diagnostic tools for assessing cardiovascular disease mostly focus on measuring a given biomarker at a specific spatial location where an abnormality is suspected. However, as a result of the dynamic and complex nature of the cardiovascular system, the analysis of isolated biomarkers is generally not sufficient to characterize the pathological mechanisms behind a disease. Model-based approaches that integrate the mechanisms through which different components interact, and present possibilities for system-level analyses, give us a better picture of a patient's overall health status.

One of the main goals of cardiovascular modelling is the development of personalized models based on clinical measurements. Recent years have seen remarkable advances in medical imaging and the use of personalized models is slowly becoming a reality. Modern imaging techniques can provide an unprecedented amount of anatomical and functional information about the heart and vessels. In this context, three-dimensional, three-directional, cine phase-contrast (PC) magnetic resonance imaging (MRI), commonly referred to as 4D Flow MRI, arises as a powerful tool for creating personalized models. 4D Flow MRI enables the measurement of timeresolved velocity information with volumetric coverage. Besides providing a rich dataset within a single acquisition, the technique permits retrospective analysis of the data at any location within the acquired volume.

This thesis focuses on improving subject-specific assessment of cardiovascular function through model-based analysis of 4D Flow MRI data. By using computational models, we aimed to provide mechanistic explanations of the underlying physiological processes, derive novel or improved hemodynamic markers, and estimate quantities that typically require invasive measurements. Paper I presents an evaluation of current markers of stenosis severity using advanced models to simulate flow through a stenosis. Paper II presents a framework to personalize a reduced-order, mechanistic model of the cardiovascular system using exclusively non-invasive measurements, including 4D Flow MRI data. The modelling approach can unravel a number of clinically relevant parameters from the input data, including those representing the contraction and relaxation patterns of the left ventricle, and provide estimations of the pressure-volume loop. In Paper III, this framework is applied to study cardiovascular function at rest and during stress conditions, and the capability of the model to infer load-independent measures of heart function based on the imaging data is demonstrated. Paper IV focuses on evaluating the reliability of the model parameters as a step towards translation of the model to the clinic. 



\section{Populärvetenskaplig Beskrivning}

Nuvarande diagnostiskverktyg för bedömning av hjärt- och kärlsjukdomar fokuserar främst på mätning av en given biomarkör vid en specifik position där abnormalitet kan förväntas. Dock, på grund av den föränderliga och komplexa naturen hos det kardiovaskulära systemet är en analys av enskilda biomarkörer i allmänhet otillräcklig för att klargöra en sjukdoms bakomliggande patologiska mekanismer. Modellbaserade metoder vilka integrerar mekanismerna genom vilka olika komponenter påverkar varandra samt möjliggör analys på systemnivå ger oss en bättre bild av patientens allmänna hälsotillstånd.

Ett av huvudmålen inom kardiovaskulär modellering är att utveckla individanpassade modeller baserade på klinisk data. Detta kan inom en snar framtid bli verklighet då stora framsteg gjorts inom medicinsk bildbehandling de senaste åren. Modern bildbehandlingsteknik kan tillgängliggöra en tidigare ouppnåelig mängd anatomisk och funktionsdata för hjärta och kärl. Det är i detta sammanhang 3-dimensionell, tidsupplöst, fas-kontrast magnetisk resonanstomografi (4D flödes-MRT) framträder som ett kraftfullt verktyg för att skapa sådana modeller. Utöver att tillhandahålla ett rikligt dataset med en enda upptagning tillåter tekniken retrospektiv analys av informationen för valfri position inom den upptagna volymen.

Denna avhandling avser att genom modellbaserad analys av 4D flödes-MRT data förbättra subjektspecifik bedömning av kardiovaskulär funktion. Genom användning av beräkningsmodeller avsåg vi att tillhandahålla mekanistiska förklaringar till de underliggande fysiologiska processerna, erhålla nydanade eller förbättrade hemodynamiska markörer samt göra uppskattningar vilka vanligtvis innebär invasiva mätmetoder. Artikel I visar en utvärdering av nuvarande biomarkörer för stenosgrad genom användning av avancerade modeller för att simulera flöde genom en stenos. Artikel II introducerar ett ramverk för att individanpassa en reducerad, mekanistisk modell av det kardiovaskulära systemet som enbart utnyttjar icke-invasiva mätmetoder såsom 4D flödes-MRT. Modelleringsmetoden kan identifiera ett stort antal kliniskt relevanta parametrar från indatan, inklusive de som representerar vänster kammares sammandragnings- och avslappningsmönster, samt tillhandahålla uppskattningar av tryck-volym loopen. I Artikel III appliceras tidigare nämnda ramverk för att studera kardiovaskulär funktion vid vila samt under träning, vilket påvisar modellens förmåga att, baserat på bilddata, tolka mätningar av hjärtats funktion oberoende av dess belastning. Artikel IV fokuserar på att bedöma tillförlitligheten av modellens parametrar som ett steg mot klinisk användbarhet. 



\section{Acknowledgements}

The work in this thesis would not have been possible without the help of many people to whom I am very grateful. First of all, I would like to thank my supervisor Tino Ebbers for his confidence, trust and guidance. His always inspiring ideas were a continuous motivation during this journey.

Special thanks go to my co-supervisors for their support and valuable input. Thanks to Jonas Lantz, for sharing his knowledge on fluid dynamics. To Petter Dyverfeldt, for his didactic explanations about turbulence mapping. To Carl-Johan Carlhäll, for fruitful discussions on physiology.

I gratefully acknowledge Matts Karlsson for his bright ideas and enthusiasm for this project. Thanks to Ann Bolger, for her insightful comments and hard work on the manuscripts. To Gunnar Cedersund, for introducing me to the field of systems biology and answering my many questions about modelling.

I would also like to thank my coworkers at the Cardiovascular Magnetic Resonance group. To Mariana Bustamante, for her positiveness, her company and for always providing me with wonderful visualizations. To Federica Viola, for her constant support, fun conversations, and for patiently analyzing many of the data in our studies. To Magnus Ziegler, for always being available for discussions and questions, and for generously proof-reading some of chapters in this thesis. To Hojin Ha, for solving the mysteries of turbulence and pressure gradients. To Sofia Kvernby, Vikas Gupta, Merih Cibis, Jonatan Eriksson, Sven Petersson, Alexandru Fredriksson and Jakub Zajac, for all the fikas, enlightening discussions, and for creating a friendly and inspiring work environment.

To Anders and Margriet, for their encouragement and support.

A Ana, Lara, Marina, Marta y Carlota, por las risas, las charlas y las salidas inesperadas.

A mis padres, mi hermana, mis tías y mi abuela, por estar siempre ahí. Por todo.

To Frank, for his patience and love. I am so lucky to have found you.

Belén Casas

Linköping, September 2018 



\section{Funding}

This work has been conducted in collaboration with the Center for Medical Image Science and Visualization (CMIV) at Linköping University, Sweden. CMIV is acknowledged for provision of financial support and access to leading edge research infrastructure. The author also acknowledges the financial support provided by:

- The European Union's Seventh Framework Programme (FP7/2007-2013) under grant 310612, project HEART4FLOW.

- The Swedish Research Council, under grant number 621-2014-6191.

- The Swedish Heart and Lung Foundation, under grant number 20140398. 



\section{List of Papers}

This thesis is based on the following papers, which will be referred to by their Roman numerals:

I. 4D Flow MRI-based Pressure Loss Estimation in Stenotic Flows: Evaluation Using Numerical Simulations.

Belen Casas, Jonas Lantz, Petter Dyverfeldt and Tino Ebbers. Magnetic Resonance in Medicine, 2016, 75, 1808-1821.

II. Bridging the Gap Between Measurements and Modelling:

A Cardiovascular Functional Avatar.

Belen Casas, Jonas Lantz, Federica Viola, Gunnar Cedersund, Ann F. Bolger, Carl-Johan Carlhäll, Matts Karlsson and Tino Ebbers.

Scientific Reports, 2017, 7, 6214.

III. Non-invasive Assessment of Systolic and Diastolic Cardiac Function During Rest and Stress Conditions Using an Integrated Image-Modelling Approach.

Belen Casas, Federica Viola, Gunnar Cedersund, Ann F. Bolger, Matts Karlsson, Carl-Johan Carlhäll and Tino Ebbers.

Submitted for journal publication, 2018.

IV. Reproducibility of 4D Flow MRI-based Personalized Cardiovascular Models; Inter-sequence, Intra-observer, and Inter-observer Variability. Belen Casas, Federica Viola, Gunnar Cedersund, Carl-Johan Carlhäll, Matts Karlsson and Tino Ebbers.

In manuscript. 
In addition, the following peer reviewed papers were published as part of the work related to this thesis:

- Assessment of Turbulent Viscous Stress Using ICOSA 4D Flow MRI for Prediction of Hemodynamic Blood Damage

Hojin Ha, Jonas Lantz, Henrik Haraldsson, Belen Casas, Magnus Ziegler, Matts Karlsson, David Saloner, Petter Dyverfeldt and Tino Ebbers

Scientific Reports, 2016, 6, 39773.

- Estimating the Irreversible Pressure Drop Across a Stenosis by Quantifying Turbulence Production Using 4D Flow MRI

Hojin Ha, Jonas Lantz, Magnus Ziegler, Belen Casas, Matts Karlsson, Petter Dyverfeldt and Tino Ebbers

Scientific Reports, 2017, 7, 46618. 


\section{Abbreviations and Nomenclature}

0D zero-dimensional

1D one-dimensional

2D two-dimensional

3D three-dimensional

4D four-dimensional

$\mathrm{A}_{\mathrm{A}} \quad$ Cross sectional area of the ascending aorta

b-SSFP balanced Steady-State Free Precession

CFD Computational fluid dynamics

E Elastance

ED End-diastole

EDP End-diastolic pressure

EDPVR End-diastolic pressure-volume relationship

EDV End-diastolic volume

EF Ejection Fraction

EOA Effective orifice area

EPI Echo-planar imaging

ES End-systole

ESPVR End-systolic pressure-volume relationship

ESV End-systolic volume

IVSD Intra voxel standard deviation

LA Left atrium 
LV Left ventricle

MAP Mean arterial pressure

MRI Magnetic Resonance Imaging

PC Phase-Contrast

PPE Pressure Poisson equation

PSF Point spread function

RF Radio-frequency

SGRE Spoiled gradient echo

SNR Signal-to-noise ratio

SV Stroke Volume

$\sigma \quad$ Standard deviation

TKE Turbulent Kinetic Energy

TPG Transstenotic pressure gradient

TPR Total peripheral resistance

u Velocity

U Mean velocity

u' Velocity fluctuation

VC Vena contracta

VENC Velocity encoding range

VTI Velocity time integral 


\section{Contents}

Abstract

Populärvetenskaplig Beskrivning vii

Acknowledgements $\quad$ ix

Funding $\quad$ xi

List of Papers $\quad$ xiii

$\begin{array}{ll}\text { Abbreviations } & \text { xv }\end{array}$

1 Introduction 1

2 Aims 3

3 Physiological Background 5

3.1 The Cardiovascular System . . . . . . . . . . . . . . . 5

3.2 Cardiac Muscle Contraction . . . . . . . . . . . . 6

3.3 The Cardiac Cycle . . . . . . . . . . . . . . . . 7

3.4 Ventricular Pressure-Volume Loops . . . . . . . . . . . . . . . . . . . . . 9

3.5 The Time-Varying Elastance . . . . . . . . . . . . . . . . . 10

3.6 Ventricular-Arterial Coupling . . . . . . . . . . . . 10

4 Cardiovascular Magnetic Resonance Imaging 13

4.1 Principles of Magnetic Resonance Imaging . . . . . . . . . . . . . 13

4.2 MRI of the Cardiovascular System . . . . . . . . . . . . . . . . . . . . . . . . . . . . . 14

4.3 Flow Measurements . . . . . . . . . . . . . . . . . . . . . . . . . 14

4.4 Turbulence Measurements . . . . . . . . . . . . . . . . 15

5 Disturbed Flow and Pressure Losses in the Cardiovascular System 17

5.1 Energy Conversion and Losses in a Stenosis . . . . . . . . . . . . . 17

5.2 Assessment of Stenosis Severity . . . . . . . . . . . . . 18

5.2.1 Pressure Gradient Estimation Using Doppler Ultrasound . . 19

5.2.2 4D Flow MRI-based Pressure Estimation . . . . . . . . . 20

6 Mathematical Modelling 2

6.1 Lumped Parameter Models . . . . . . . . . . . . . . . . . . . . . . 24

6.2 Mathematical Formalism . . . . . . . . . . . . . . . . . . 25

6.3 Model Personalization . . . . . . . . . . . . . . 26

6.3.1 Parameter Estimation . . . . . . . . . . . . . . 27

6.4 Parameter Uncertainty and Identifiability Analysis . . . . . . . . . . 28

6.4.1 Parameter Confidence Intervals _ . . . . . . . . . . . 28 
6.4 .2 Identifiability Analysis . . . . . . . . . . . . . . . 29

7 Methods and Results $\quad 31$

7.1 Accuracy of MRI-based Methods for Estimating the Irreversible

Pressure Drop . . . . . . . . . . . . . . . . . . . 31

7.1 .1 Numerical Flow Data . . . . . . . . . . . . . 32

7.1 .2 PC-MRI Simulations . . . . . . . . . . . . . . 32

7.1.3 Results and Interpretation . . . . . . . . . . . 33

7.2 Model Personalization Using 4D Flow MRI . . . . . . . . . . . . 35

7.2.1 The Cardiovascular Model . . . . . . . . . . . . . . . 35

7.2 .2 Personalization Framework . . . . . . . . . . . . 38

7.2 .3 Model Evaluation . . . . . . . . . . . . . . . . 39

7.3 Model-based Assessment of Cardiac Function During Rest and Stress

Conditions . . . . . . . . . . . . . . . . . 42

7.3.1 Cardiovascular Indices and Flow Measurements . . . . . . . 42

7.3.2 Time-varying Elastance Parameters . . . . . . . . . . . . 46

7.4 Reproducibility of Subject-Specific Model Parameters . . . . . . . 46

7.4.1 Variability in the Input Measurements . . . . . . . . . . . . . 47

7.4.2 Intra- and Inter-observer Variability . . . . . . . . . . . . . . . . . . . 47

7.4 .3 Inter-sequence Variability . . . . . . . . . . . . . . 48

8 Discussion and Future Directions $\quad 51$

8.1 Contributions of this Thesis . . . . . . . . . . . . . 51

8.2 Estimation of The Irreversible Pressure Drop . . . . . . . . . . . 52

8.3 Creating personalized models using 4D Flow MRI . . . . . . . . . . 53

8.3.1 Image-Model Fusion . . . . . . . . . . . . . . . . . . 53

8.3.2 Model Evaluation . . . . . . . . . . . . . . . 54

8.4 Future Directions . . . . . . . . . . . . . . . . . 55 


\section{Chapter 1}

\section{Introduction}

Cardiovascular function is extremely complex, with multiple physiological processes acting towards achieving a common goal: the delivery of blood flow to all parts of the body. The pumping function of the heart is influenced by the interaction with the vascular system, responding to oxygen demands and changes in loading conditions. As a result of these interactions, cardiovascular diseases are generally not restricted to a specific site, but instead manifest themselves at several locations in the heart and the vessels. In this context, the task of unraveling the mechanisms behind disease based solely on experimental measurements becomes increasingly challenging.

Computational modelling has been proposed as a tool to better understand cardiovascular function in health and disease. Although the use of computational models for studying hemodynamics has a long history, it is only in recent years that the field has seen major progress. A main reason for this progress is the development of advanced imaging techniques, which allow for the incorporation of an increasing variety and quantity of information into the models. With current imaging techniques, it is possible to measure the heart's anatomy and motion, myofiber architecture, blood flow and even metabolism [1]. Three-dimensional, time-resolved phase-contrast MRI with three-directional velocity-encoding, usually referred to as 4D Flow MRI, is one such technique that allows investigation of the cardiovascular system in great detail. 4D Flow MRI provides velocity information with volumetric coverage of the regions of interest and permits retrospective assessment of the data at any location within the acquired volume [2].

In this work, we focus on leveraging the advanced data obtained with 4D Flow MRI using personalized models of the cardiovascular system. By doing so, we aim to obtain information on hemodynamics that is not feasible to measure, and derive biomarkers for better assessment of cardiovascular function. Translation of models into clinical care is challenging, and requires exhaustive evaluation in order to gain confidence in their predictive capabilities. However, once they are incorporated into clinical routine, computational models have the potential to make a major impact on patient care by assisting in the diagnosis of diseases and adding to the process of treatment planning. 



\section{Chapter 2}

\section{Aims}

The goal of the research described in this thesis was to improve non-invasive assessment of cardiovascular function by integrating computational models with data from 4D Flow MRI acquisitions. Specifically, we focused on the following aims:

- Using advanced computational models to evaluate the accuracy of current 4D Flow MRI-based methods for estimation of the pressure drop over a stenosis.

- Developing a framework that combines non-invasive measurements, including 4D Flow MRI data, and a mechanistic, lumped parameter model of the cardiovascular system to obtain personalized parameters characterizing global hemodynamics.

- Utilizing the modelling approach to investigate cardiac hemodynamics at rest and during stress.

- Investigating the reliability of the model parameters with respect to variability in the analysis of the MRI input measurements. 



\section{Chapter 3}

\section{Physiological Background}

This section serves as an introduction to cardiovascular physiology and the physical properties of the heart. For a more detailed perspective, the reader is referred to literature such as the works by Guyton and Hall [3] or Katz [4].

\subsection{The Cardiovascular System}

The primary role of the cardiovascular system is to drive, control, and maintain blood flow to the different organs of the body. It comprises the heart and the vascular system. Through complex mechanisms, the cardiovascular system is able to adapt to changing demands during everyday life and provide the necessary blood flow to the tissues. These demands can vary considerably with changes due to exercise, body position, or intrathoracic pressure.

The vascular system consists of a branching network of blood vessels, whose characteristics are determined by the function they perform. Large, elastic arteries form a distribution network that carries blood away from the heart. The veins, on the other hand, act as a collection system and transport deoxygenated blood from the tissues back to the heart. Bridging in-between the arteries and veins is a highly branched network of small vessels referred to as microcirculation, that allows diffusion of substances between the blood and the body tissues.

The heart serves as a pump to propel blood throughout the vascular system. Functionally, it can be divided into two simultaneously acting pumps: the left heart and the right heart. Both pumps are composed of two chambers, one smaller, known as the atrium, and one larger, known as the ventricle. The atria behave mainly as passive reservoirs for venous return to the heart and contract to aid ventricular filling. The ventricles then provide the main force that is required to propel the blood through the vascular system, down to the peripheral vessels. The left ventricle supplies the systemic circulation with oxygenated blood, while the right ventricle pumps oxygen-depleted blood through the pulmonary circulation for oxygenation. The atrioventricular valves (i.e. the mitral and tricuspid valves) connect the atria with the ventricles. Likewise, the semilunar valves connect the ventricles with their corresponding arterial outflow tracts. The aortic valve is located between the left ventricle and the aorta, and the pulmonary valve connects the right ventricle with the pulmonary artery. The heart valves open and close as a result of changes in 


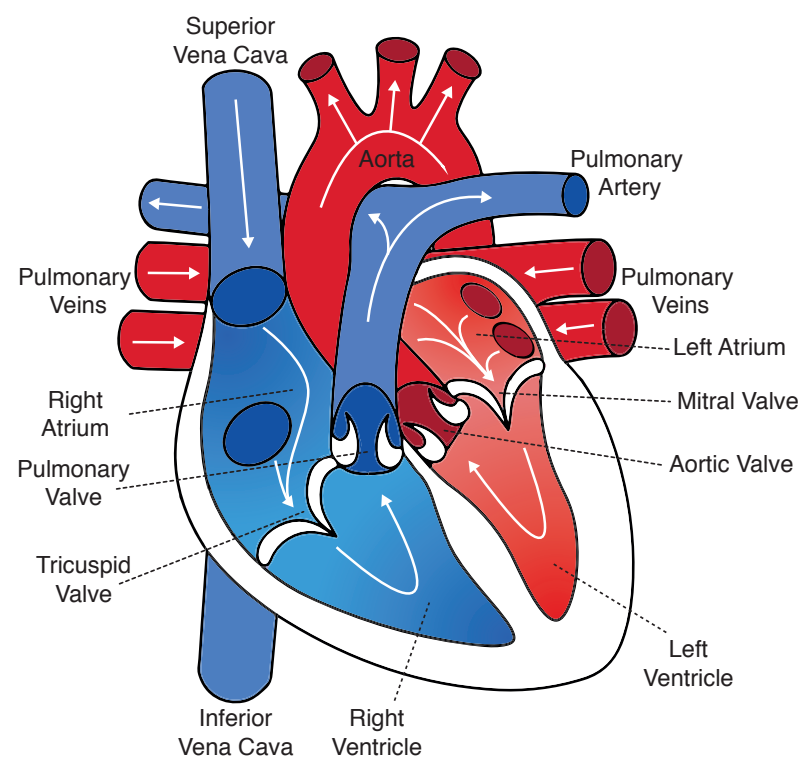

Figure 1: Schematic diagram of the heart. The left side of the heart containing oxygenated blood is shown in red and the right-sided heart containing deoxygenated blood is depicted in blue. The arrows indicate the course of blood flow through the chambers and the valves.

pressure due to the contraction and relaxation of the heart, and prevent backflow during normal conditions [3]. A detailed image of the heart is shown in Figure 1.

\subsection{Cardiac Muscle Contraction}

The contractile properties of the ventricle are determined by the ability of cardiac muscle cells, known as myocytes, to shorten and generate force. Shortening of the myocytes is triggered by an action potential, which depolarizes the cell membrane and allows the inflow of $\mathrm{Ca}^{2+}$ ions into the sarcoplasm. The $\mathrm{Ca}^{2+}$ inward current elicits a series of mechanisms that lead to actin-myosin cross-bridge formation (i.e. excitation-contraction coupling) allowing contraction of the heart [5]. The excitation-contraction coupling process is crucial for the contraction and relaxation of the heart's chambers, and abnormalities in $\mathrm{Ca}^{2+}$ handling are a known cause of both contractile dysfunction and arrhythmias [6].

In addition to $\mathrm{Ca}^{2+}$ handling, contractility is influenced by the length of relaxed cardiac muscle fibers. During diastole, muscle fibers are highly compliant (i.e. low stiffness) and generate low tension as they are stretched. During systole the cardiac muscle becomes much stiffer and the applied force reaches a maximal value. This systolic force increases considerably for increasing lengths of the relaxed muscle fibers. The maximal force corresponds to an optimal initial length that allows for 
a maximum number of actin-myosin cross-bridges [7]. For lengths larger than the optimal, the force that can be generated by the muscle fibers reaches a plateau because the overlap between the actin and the myosin fibers decreases [8].

Cardiac muscle fibers are arranged in bundles wrapping around the heart in a helical pattern. The forces generated by the fibers are translated into pressures within the ventricle and are proportional to the EDV, which determines the relaxed length of the fibers. Therefore, pressures and volumes in the ventricle, as represented by the pressure-volume loop, are interconnected to muscle force-length relationships and have various common features [9].

\subsection{The Cardiac Cycle}

The cardiac cycle refers to the succession of events that take place in one heart beat. It can be divided into two major periods, systole and diastole. The ventricles contract during systole and achieve their maximal contractility at end-systole, subsequently relaxing during diastole. The following discussion will focus on the hemodynamic events related to the left heart. These events are similar for the right heart, although right ventricular pressures are lower than those in the left ventricle.

The systolic and diastolic periods can be further divided into different phases, based on the contractile state of the chambers and the position of the valves. These phases can be illustrated using a Wiggers diagram, as shown in Figure 2. In this diagram, the pressure and volume traces of the left ventricle are represented as a function of time. A schematic diagram of the electrocardiogram (ECG) is included to illustrate the relation between the electrical and the mechanical events along the cardiac cycle. The following phases are represented:

- Atrial contraction: Atrial contraction is initiated by depolarization in the atrium (P wave in the ECG). This causes an increase in atrial pressure, aiding the flow of blood into the ventricle. This period is also referred to as the late filling phase of the ventricle. At the end of atrial contraction, the ventricular volume is at its maximum and corresponds to the end-diastolic volume (EDV). The end of atrial contraction coincides with the end of ventricular relaxation.

- Isovolumic contraction: Ventricular depolarization, represented by the QRS complex in the ECG, causes ventricular contraction. The pressure in the ventricle rises slightly and eventually exceeds that in the atrium, leading to closure of the mitral valve. Since both the mitral and the aortic valves are closed, the ventricular volume remains constant. Therefore, the ventricle contracts isovolumically and the ventricular pressure increases rapidly. When the ventricular pressure rises above the aortic pressure, the aortic valve opens and the ejection phase starts.

- Ventricular ejection: Following the opening of the aortic valve, the ventricle ejects blood into the aorta and the ventricular volume decreases. The ventricular and aortic pressure curves are determined by the interaction between 


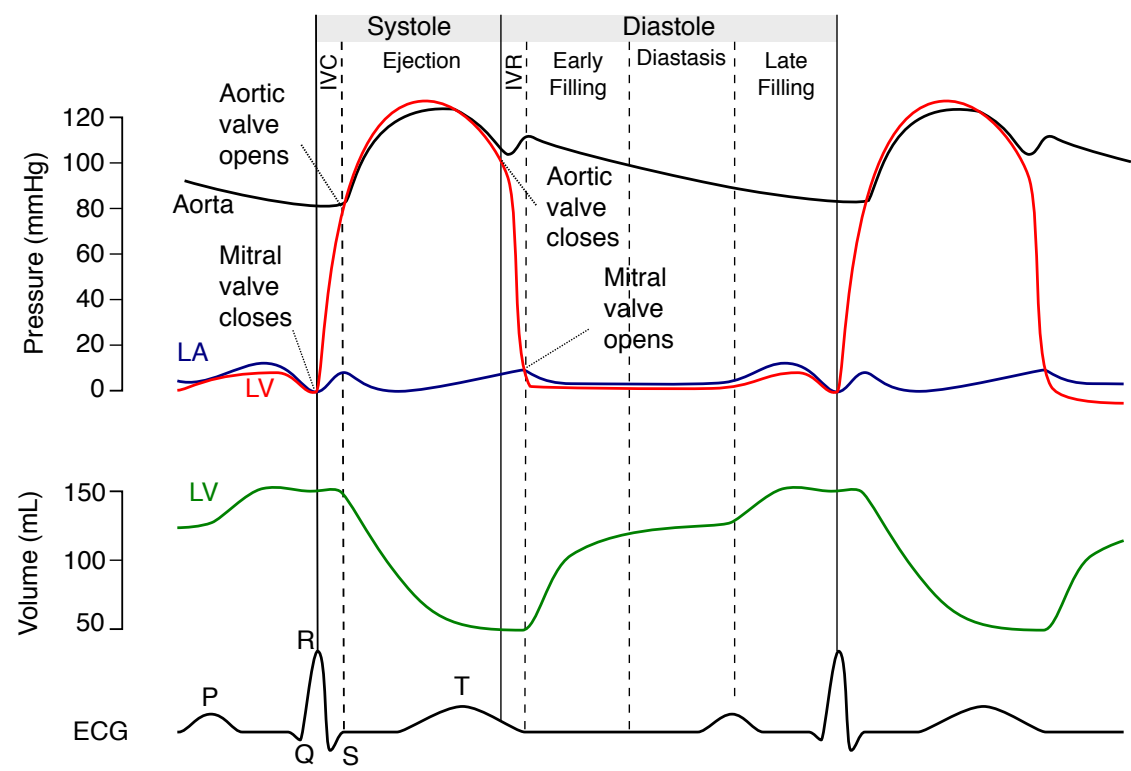

Figure 2: Wiggers diagram illustrating the events in left cardiac function during the cardiac cycle. The diagram shows changes in pressures and left ventricular volumes, and their relation with the electrocardiogram. ECG, electrocardiogram; LA, left atrium; LV, left ventricle; IVC, isovolumic contraction; IVR, isovolumic relaxation

the changes in ventricular contractility and the properties of the arterial system. In particular, the curves reflect the relation between the ejection rate of flow into the aorta and the rate at which flow is distributed throught the arterial tree. Based on the shape of the aortic curve, the ejection phase can be subdivided into two phases: rapid ejection, and decreased ejection. During the rapid ejection phase, arterial pressure continues to increase as the rate of ventricular ejection into the aorta exceeds the rate of flow out to the tissues. The increase in outflow to the tissues in relation to inflow from the ventricle causes the decrease in pressure along the decreased ejection phase [4]. During the decreased ejection phase, the ventricle starts to repolarize, as evidenced by the T wave in the ECG. The blood volume remaining in the ventricle at the end of ejection is the end-systolic volume (ESV).

- Isovolumic relaxation: As repolarization continues, the ventricle relaxes and ventricular pressure decreases. This causes backflow from the aorta towards the ventricle, leading to the closure of the aortic valve. With the mitral and the aortic valves closed, the ventricle relaxes at a constant volume. The ventricular pressure declines rapidly, and when it drops below atrial pressure the mitral valve opens. 
- Ventricular filling: Opening the mitral valve allows rapid inflow of blood into the ventricle, as indicated by the increase in ventricular volume. This period, referred to as the early rapid filling phase, is where the majority of ventricular filling takes place. After rapid filling, the pressures in both the atrium and the ventricle increase slightly, and the pressure gradient across the mitral valve is minimal. Hence, there is reduced flow from the atrium to the ventricle and this corresponds to the period of reduced filling or diastasis. The length of diastasis is influenced by the heart rate, being progressively reduced at increasing heart rates as the early and late filling phases begin to merge [10]. Following diastasis, atrial depolarization triggers the contraction of the atrium and a new cardiac cycle begins.

Systole can be defined as the time interval between closure of the mitral valve and closure of the aortic valve, and includes the isovolumic contraction phase and the ejection phase. Diastole corresponds to the period in which the relaxation and filling of the ventricle occur, from isovolumic relaxation to the end of the late filling phase.

\subsection{Ventricular Pressure-Volume Loops}

The investigation of cardiac function based on pressure-volume loops was initiated by Otto Frank in 1895. With his experiments on isolated frog hearts, Frank demonstrated that ventricular pressure rose as the end-diastolic volume increased [11]. Starling et al. performed experiments on anesthetized dogs and observed that increasing end-diastolic pressure yielded an increase in cardiac output [12]. These observations led to the well-known Frank-Starling law of the heart, which states that cardiac performance, in terms of the ability of the ventricle to generate pressure or eject blood, is enhanced as preload increases.

Pressure-volume loops show the instantaneous relationship between intraventricular pressure and volume through the cardiac cycle. A schematic of a pressurevolume loop can be seen in Figure 3A, where the different phases of the cardiac cycle are illustrated. The dynamic changes in the stiffness of the ventricle throughout the cardiac cycle can be derived from this representation. In each cycle the muscle fibers relax and contract, changing the stiffness of the ventricle. The minimal and maximal stiffness correspond to the times of end-diastole and end-systole, respectively. During diastole the passive mechanical behaviour of the ventricle is typically characterized by its compliance. The compliance $(\mathrm{C})$ is defined as the change in volume for a given change in pressure, as given by equation (1).

$$
\mathrm{C}=\frac{\Delta V}{\Delta P}
$$

where $V$ represents the volume, and $P$ the pressure.

During diastolic filling, the ventricle is highly compliant and fills at low pressures. The relation between pressure and volume follows an exponential curve [13], indicating that the ventricle becomes stiffer as the volume increases. This exponential 
curve is known as the end-diastolic pressure-volume relationship (EDPVR) or the passive filling curve of the ventricle. As the EDPVR is non-linear, the compliance of the ventricle changes with volume during diastole [14].

A similar relationship can be obtained at end-systole. Suga et al. [15] reported that end-systolic points on pressure-volume loops obtained from the same heart under different loading conditions followed an approximately linear relationship. This relationship is referred to as the end-systolic pressure-volume relationship (ESPVR). The slope of the ESPVR, the end-systolic or maximal elastance $\left(\mathrm{E}_{\max }\right)$, has been proposed as an index of the contractility of the ventricle [16]. An advantage of $\mathrm{E}_{\max }$ compared to other indexes to assess $\mathrm{LV}$ systolic function, such as ejection fraction (EF), is that it is independent of loading conditions [15] and therefore constitutes a more accurate measure of the actual contractility of the heart. The EDPVR and the ESPVR of the left ventricle are illustrated in Figure 3B.

\subsection{The Time-Varying Elastance}

So far, we have considered the pressure-volume relationships at end-diastole and end-systole. However, such relationships can be extracted at any time point along the cardiac cycle. Isochrones are defined as the lines that connect instantaneous pressure-volume points obtained under different loading conditions at a given time. The slope of each isochrone defines the elastance of the ventricle at that specific time point. As seen in Figure 3C, the slopes change during the cardiac cycle, gradually increasing during systole and decreasing during diastole. These changes in time lead to the concept of time-varying elastance $E(t)$, which was first introduced by Suga and Sagawa [16]. $E(t)$ describes the instantaneous relationship between left ventricular pressure $P(t)$ and ventricular volume $V(t)$, as given by:

$$
\mathrm{E}(\mathrm{t})=\frac{P(t)}{V(t)-V_{0}}
$$

where $V_{0}$ is the unstressed volume of the heart. $E(t)$ increases during systole until it reaches its maximal value $\mathrm{E}_{\max }$, and subsequently decreases during diastole, returning to the passive elastance value $\mathrm{E}_{\min }$ (Figure 3D). The time-varying elastance has been shown to be load independent, and sensitive to alterations in the inotropic state of the ventricle [17, 18].

\subsection{Ventricular-Arterial Coupling}

Changes to the loading conditions of the ventricle are reflected in the appearance of the pressure-volume loop. Preload refers to the stretch of the cardiac muscle fibers at the end-diastole, prior to the onset of ejection. As previously described, the force generated by the ventricle depends on the initial length of the muscle fibers. Common measures of preload include the EDV and the end-diastolic pressure (EDP). 

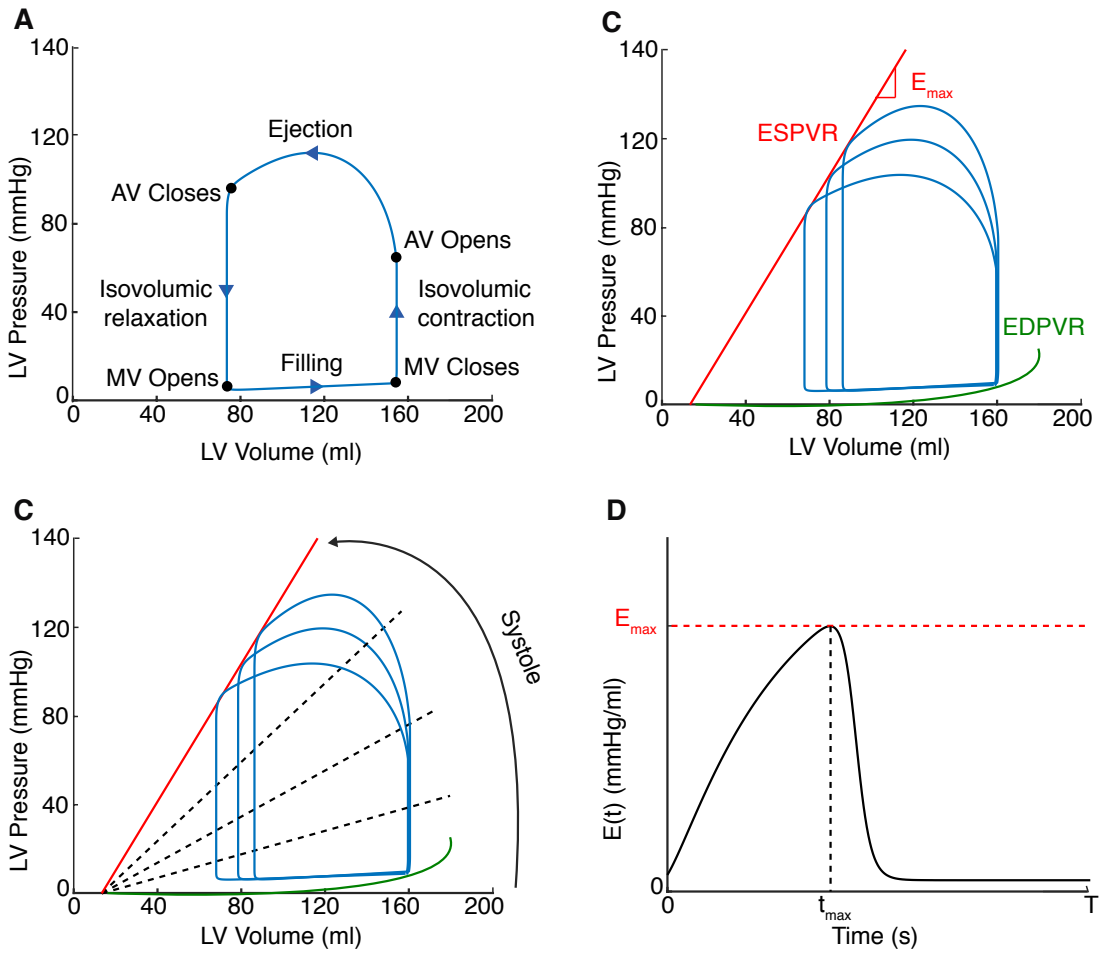

Figure 3: Overview of the pressure-volume loop and its relationship to the time-varying elastance function. A: An example of a pressure volume loop representing changes in intraventricular volume and pressure during the cardiac cycle. B: End-systolic and enddiastolic pressure volume relationships (EDPVR and ESPVR, respectively) obtained from a family of pressure-volume loops. The slope of the ESPVR is the maximal elastance of the ventricle $\left(\mathrm{E}_{\max }\right)$. $\mathrm{C}$ : Examples of time-varying isochrones during the cardiac cycle. D: Time varying elastance function. $t_{\max }$, time instant corresponding to the maximal elastance $E_{\max }$.

The concept of afterload is related to the load that the ventricle must overcome during ejection. In the absence of valvular pathologies, such as mitral regurgitation or aortic stenosis, this afterload is mainly determined by the properties of the arterial system. Indices of afterload include aortic pressure, arterial impedance, and the total peripheral resistance (TPR), which is defined as the ratio between the mean arterial pressure (MAP) and the mean flow in the arterial system (i.e. the cardiac output (CO)).

In addition to the work by Frank and Starling [11], other authors have performed experiments to study the effects of preload and afterload under varying conditions. Weber et al. [19] investigated the effects of afterload and reported a linear decrease in stroke volume with increasing end-systolic arterial pressure. The concept of ESPVR can also be used to illustrate the effects of changes in preload and afterload. As 
the end-systolic points of the pressure-volume loops for varying loading conditions lie on the same ESPVR, an increase in preload (EDV) results in an increased SV. Similarly, an increase in afterload translates into a decrease in end-systolic volume (ESV), and therefore a lower SV. However, in the following cardiac cycle, the ventricle will adapt to the increased afterload in order to maintain the initial SV. This is accomplished by increasing the EDV, shifting the pressure-volume loop to the right. Such adaptation implies an increase in the pressure-volume area and therefore involves greater mechanical work [20]. 


\section{Chapter 4}

\section{Cardiovascular Magnetic Resonance Imaging}

\subsection{Principles of Magnetic Resonance Imaging}

This section aims to provide an overview of the principles behind Magnetic Resonance Imaging. For an extended description, the reader is referred to [21-23]. Magnetic Resonance Imaging exploits the magnetic properties of the hydrogen nucleus, the most abundant one within biological tissues, to create contrast in an image. Hydrogen nuclei generate a magnetic field as they move, and can be regarded as small magnets or "spins". When the spins are exposed to an external magnetic field $B_{0}$, they start to precess about the field's axis. The angular frequency of this precession $\left(\omega_{0}\right)$, referred to as Larmor frequency, is determined by the Larmor equation:

$$
\omega_{0}=\gamma B_{0}
$$

where $\gamma$ is the gyromagnetic ratio. The gyromagnetic ratio for the hydrogen nucleus is approximately $42.6 \mathrm{MHz} / \mathrm{T}$. This results in a Larmor frequency around $64 \mathrm{MHz}$ in a magnetic field of $1.5 \mathrm{~T}$.

At this equilibrium state, the net magnetization of the spins $(M)$ is aligned with $B_{0}$ and is too small to be measured. The orientation of $M$ needs to be manipulated in order to obtain a measurable signal. This is achieved with radio-frequency (RF) pulses. If a RF pulse matched at the Larmor frequency is applied, $M$ is flipped from the longitudinal plane to the transverse plane. The flip angle $(\alpha)$ depends on the duration and the intensity of the RF pulse. A flip angle $\alpha=90^{\circ}$ implies that $M$ is flipped completely onto the transverse plane. Since only the transverse component of $M$ is detectable, $\alpha=90^{\circ}$ produces the strongest signal.

When the RF pulse is switched off, the spins return to the equilibrium state through a process known as relaxation. The time it takes for the spins to relax conveys information on the tissues in which they are located. Two types of relaxation times characterize the relaxation process. The spin-lattice (longitudinal) relaxation time (T1) describes the recovery of $M$ along the direction of $B_{0}$ as a result of the transfer of energy from the spins to their surrounding environment ("lattice"). The spin-spin (transversal) relaxation time (T2) characterizes the decay in the transverse 
component of $M$ due to the interaction between the magnetic fields of neighboring spins.

Image generation in MRI is accomplished through spatial encoding. Spatial encoding consists of applying a sequence of magnetic gradients that allow selective excitation of the spins within the object being imaged. These gradients cause the magnetic field to vary linearly as a function of position. Since the relationship between the Larmor frequency of the spins and the gradient field is known, it is possible to infer spatial information from the signals detected by the receiver coil in the MRI system. Image reconstruction involves Fourier Transformation of the detected signals, in order to decode the magnitude and the spatial locations of the frequency components. The result is an image in which contrast is determined by the relaxation properties of the imaged tissues.

\subsection{MRI of the Cardiovascular System}

Cardiovascular Magnetic Resonance is a versatile imaging modality that permits comprehensive evaluation of cardiac anatomy and structure, tissue characteristics, myocardial perfusion and blood flow. For these reasons, it is increasingly being considered as a "one-stop shop" for the assessment of cardiovascular function [24] and has become the imaging modality of choice in many cardiac modelling studies. The following sections describe the MRI acquisition techniques relevant for this thesis.

\subsection{Flow Measurements}

The most common MRI technique for measuring flow velocity is phase-contrast MRI (PC-MRI) [25]. PC-MRI relies on the phase shift accumulated by moving flow spins under the influence of a bipolar gradient. The phase shift $\phi$ accumulated by the spin is proportional to its velocity. Under the assumption that the distribution of velocities within the voxel is symmetric about the mean velocity $(U)$, the relation between $\phi$ and $U$ can be described as:

$$
\phi=k_{v} U+\phi_{0}
$$

where $k_{v}$ describes the amount of phase motion sensitivity and $\phi_{0}$ represents an additional phase shift due to inhomogeneities in the magnetic field $B_{0}$. To eliminate $\phi_{0}$, a set of data with a different motion sensitivity can be acquired. The corrected $\phi_{0}$ is then computed as the difference between these two datasets.

The range of velocities that can be measured with PC-MRI is determined by the velocity encoding (VENC) parameter. The VENC value defines the velocity that corresponds to a phase shift of $\pm \pi$ radians. For velocity values outside the range \pm VENC, the phase exceeds $\pm \pi$, leading to an incorrect mapping of this velocities within the interval \pm VENC (i.e. aliasing). Choosing an appropiate VENC value is 
critical for accurately measuring the velocities, and this choice should be based on a priori knowledge of the highest expected velocity in the region to be imaged.

\section{D Flow MRI}

4D Flow MRI can be described as phase-contrast MRI with flow encoding in three spatial directions that is resolved relative to time and all three dimensions of space $[2,26,27]$. The acquired dataset comprises four four-dimensional $(4 \mathrm{D}=3 \mathrm{D}+$ time) volumes: a magnitude volume, depicting anatomy, and three velocity volumes representing the velocities in three orthogonal spatial directions $\left(V_{x}, V_{y}\right.$ and $\left.V_{z}\right)$. The data are acquired over several heart beats and retrospectively sorted to generate datasets corresponding to one cardiac cycle.

The wealth of data available within a single acquisition and the possibility to perform retrospective analysis make 4D Flow MRI a valuable tool for investigating cardiovascular flow in vivo. Several studies have applied this technique to visualize and quantify flows in the ventricles [28-32], the atria [33, 34], the heart valves [35, 36] and the aorta [37-39]. Furthermore, a number of advanced hemodynamic parameters can be derived from the 4D Flow MRI data, such as turbulent kinetic energy (TKE) [40, 41], pressure differences [42, 43], wall shear stress [44, 45] and pulse wave velocity $[45,46]$. Most of these parameters can not be obtained using other currently available imaging techniques.

\subsection{Turbulence Measurements}

The flow measurements obtained with PC-MRI correspond to the mean velocity field. In turbulent flow, however, the velocity $(u)$ includes both a mean and a fluctuating component ( $U$ and $u^{\prime}$, respectively). For an arbitrary spatial direction $i$, this can be described as:

$$
u_{i}=U_{i}+u_{i}^{\prime} \quad\left(\mathrm{ms}^{-1}\right)
$$

The turbulent intensity in each direction $i\left(\sigma_{i}\right)$ is defined as the standard deviation of the fluctuating component:

$$
\sigma_{i}=\sqrt{\overline{u_{i}^{\prime 2}}} \quad\left(\mathrm{~ms}^{-1}\right)
$$

While the mean velocity $U_{i}$ can be accurately measured with PC-MRI, resolving the fluctuating component would require values of temporal and spatial resolution that are not feasible. However, turbulence intensity can be measured using an extension of the PC-MRI technique referred to as intravoxel velocity standard deviation (IVSD) mapping, or turbulence mapping [40, 47, 48]. This approach exploits the effects of turbulence on the magnitude of the PC-MRI signal. In turbulent flow, application of a bipolar gradient will result in attenuation of the PC-MRI signal magnitude. This attenuation is caused by the presence of a velocity distribution within the voxel, and its value depends on the characteristics of the bipolar gradient 
and the standard deviation of the velocity distribution [49]. The magnitude of the PC-MRI signal can then be used to estimate the IVSD, which is a measure of the turbulence intensity $\left(\sigma_{i}\right)$. Assuming a gaussian distribution of velocities within the voxel, the IVSD can be calculated as:

$$
\sigma_{i}=\frac{1}{k_{v}} \sqrt{2 \ln \left(\frac{\left|S_{i}(0)\right|}{\left|S_{i}\left(k_{v}\right)\right|}\right)} \quad\left(\mathrm{ms}^{-1}\right)
$$

where $S_{i}(0)$ is the signal acquired at zero motion sensitivity and $S_{i}\left(k_{v}\right)$ is the signal acquired at motion sensitivity $k_{v}$.

The variance of the velocities in each direction can be regarded as normal stresses and constitute the diagonal of the Reynolds stress tensor $(\mathbf{R})$ :

$$
\mathbf{R}=\rho \overline{u_{i}^{\prime} u_{j}^{\prime}} \sim \rho\left(\begin{array}{lll}
\overline{u_{1}^{\prime 2}} & \overline{u_{1}^{\prime} u_{2}^{\prime}} & \overline{u_{1}^{\prime} u_{3}^{\prime}} \\
\overline{u_{2}^{\prime} u_{1}^{\prime}} & \overline{u_{2}^{\prime 2}} & \overline{u_{2}^{\prime} u_{3}^{\prime}} \\
\overline{u_{3}^{\prime} u_{1}^{\prime}} & \overline{u_{3}^{\prime} u_{2}^{\prime}} & \overline{u_{3}^{\prime 2}}
\end{array}\right)
$$

where $\rho$ is the fluid density. The Reynolds stress tensor is a second-order symmetric velocity tensor, where the diagonal components are normal stresses and the off-diagonal components correspond to shear stresses.

The (IVSD) mapping approach allows estimation of normal stresses. The shear stresses can in principle be estimated by performing measurements in additional encoding directions, but this estimation has proven difficult because of SNR issues [50]. To mitigate the effect of noise on the estimations, a technique based on a six-directional encoding scheme referred to as ICOSA6 has been proposed recently [51]. The implications of this technique in relation to the derivation of pressure gradients using 4D Flow MRI are discussed in section 8. 


\section{Chapter 5}

\section{Disturbed Flow and Pressure Losses in the Cardiovascular System}

Blood flow in the healthy cardiovascular system is primarily laminar, with wellordered fluid layers that slide in parallel. The presence of altered flow patterns, on the other hand, is generally associated with disease. Stenotic valves and vessels are examples of pathologies which are known to disrupt the normal characteristics of blood flow, causing turbulence and complex flow patterns such as enhanced helical and vortical blood flow formations in the post-stenotic region [36, 52-56]. This leads to pressure losses resulting in an increased cardiac afterload that may eventually lead to heart failure if the pathology is left untreated [57].

This chapter aims to review the concepts of energy and pressure losses when applied to evaluate stenotic valvular disease, and give an overview of current echocardiography and MRI-based approaches for non-invasive quantification of transstenotic pressure gradients.

\subsection{Energy Conversion and Losses in a Stenosis}

Figure 4 illustrates the changes and losses in mechanical energy that take place across a stenosis. Neglecting the effect of gravity, the total energy of the flow can be described as the sum of static pressure (potential energy) and kinetic energy. Flow accelerates in the contraction region (points 1 to 2), resulting in an increase in kinetic energy at the expense of a drop in pressure. The velocity gradually increases until it reaches its maximum at the vena contracta (VC), which corresponds to the narrowest part of the velocity jet (point 2). The pressure drop at the vena contracta is referred to as the maximum transstenotic pressure gradient $\left(T P G_{\text {max }}\right)$. The energy losses in the contraction section of the stenosis are negligible and mainly due to laminar viscous dissipation [58]. Therefore, this gradient is not representative of energy losses in the flow.

In the post-stenotic region (points 2 to 3), part of the kinetic energy is reconverted into pressure as flow decelerates. This phenomenon, known as pressure recovery, is a recognized source of discrepancy between catheter measurements of the transstenotic pressure gradient and Doppler echocardiography-based estimations using peak velocities at the VC [59-65]. The pressure gradient following pressure 


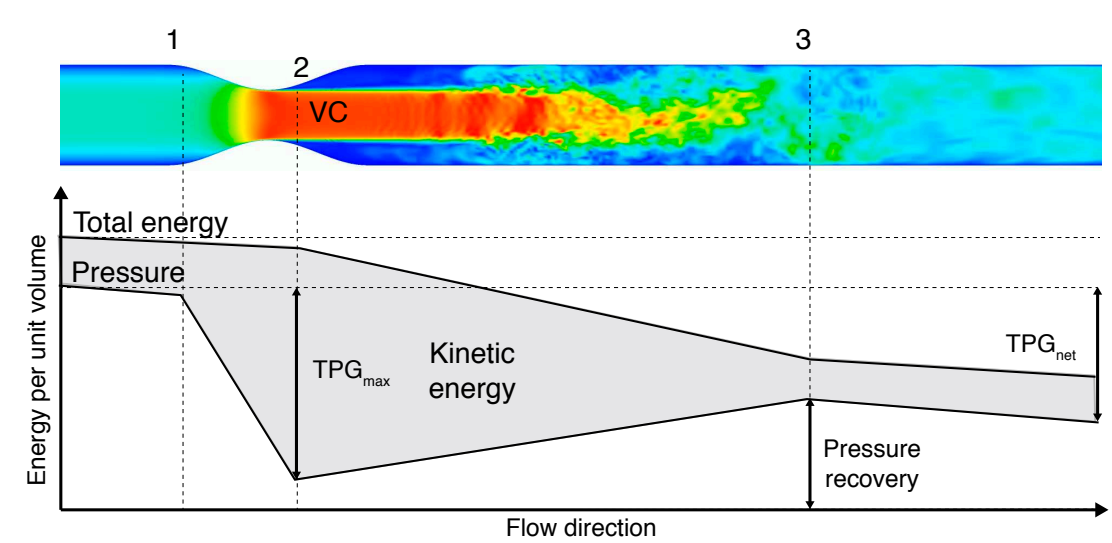

Figure 4: Schematic representation of a stenosis with the corresponding changes in pressure and total energy. The gray area represents the kinetic energy. The flow is visualized as a snapshot of the velocity in a constricted pipe from a computational fluid dynamics (CFD) simulation. $T P G_{\max }$, maximum transstenotic pressure gradient; $T P G_{n e t}$, net transstenotic pressure gradient; $\mathrm{VC}$, vena contracta.

recovery is referred to as irreversible pressure drop (net transstenotic pressure gradient, $\left.T P G_{n e t}\right) . T P G_{n e t}$ reflects the increase in left ventricular afterload due to flow inefficiency and is a proven clinical predictor of adverse outcome in aortic stenosis patients [66].

The amount of kinetic energy that is not reconverted into pressure is lost mainly due to turbulence and, to a much lesser extent, laminar viscous dissipation [67]. In turbulent flow, energy transfer occurs in a cascade, as illustrated in Figure 5. The energy in the large eddies is passed to smaller eddies, which in turn pass this energy to progressively smaller eddies in a fractal manner. Energy dissipation into heat occurs at the smallest eddies, where the effect of viscosity is increased. It should be noted that both viscous dissipation losses and turbulent losses are due to viscous friction [68].

Besides largely contributing to pressure loss, the stresses developed in turbulent flow may induce mechanical damage to blood constituents, increasing the risk for hemolysis as well as platelet activation and thrombus formation $[69,70]$.

\subsection{Assessment of Stenosis Severity}

The gold standard for measuring the net transstenotic pressure gradient $T P G_{\text {net }}$ is cardiac catheterization, but this procedure is invasive and therefore its application in clinical routine is limited. Several approaches have been proposed to estimate transstenotic pressure gradients non-invasively using measurements from either echocardiography or MRI. The following is a summary of the methods relevant for this thesis. 


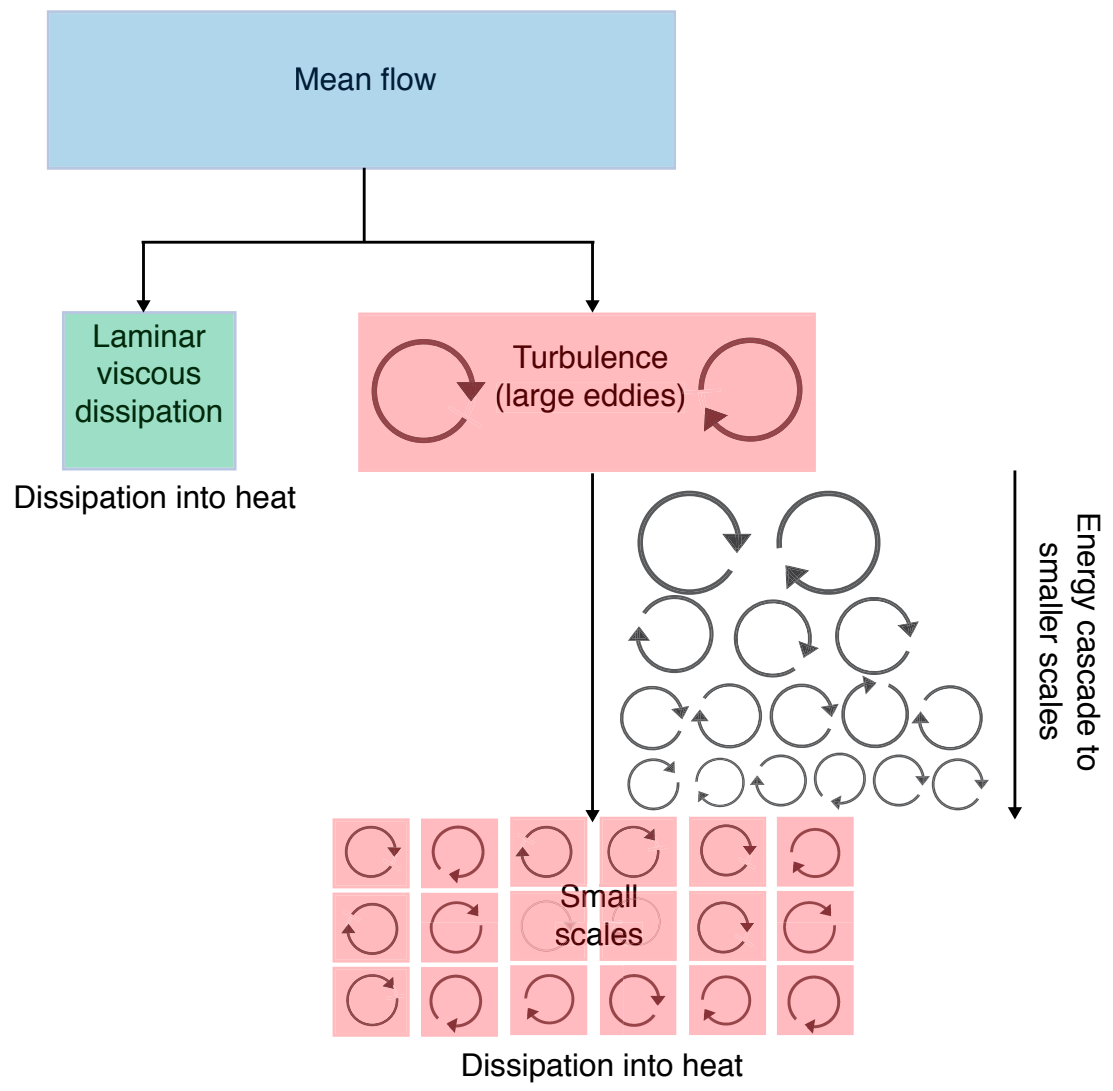

Figure 5: Schematic diagram representing conversion and dissipation of energy in a stenosis. The kinetic energy in the mean flow is mostly transferred to large turbulent eddies and, to a lesser degree, lost due to laminar viscous dissipation into heat. The kinetic energy in turbulent flow is transferred in a cascade, from large to smaller eddies until it is dissipated into heat.

\subsubsection{Pressure Gradient Estimation Using Doppler Ultrasound}

\section{Simplified Bernoulli}

In clinical practice, Doppler echocardiography is commonly used to assess pressure gradients non-invasively [71]. The maximum transstenotic pressure gradient $T P G_{\text {max }}$ is estimated based on the Bernoulli principle using Doppler measurements of the peak transvalvular velocity $v_{\max }$ (i.e. peak velocity in the vena contracta), assuming that the velocity proximal to the stenosis can be disregarded [72]:

$$
T P G_{\max }=4 v_{\max }^{2} \quad(\mathrm{mmHg})
$$


where the velocity $v_{\max }$ is given in $\mathrm{m} / \mathrm{s}$. Although Doppler techniques can reliably measure the peak velocity of the jet, these measurements are limited to a single direction determined by the ultrasound beam and the accuracy depends, among other factors, on the correct alignment between the flow jet and the probe [71, 73, 74]. More importantly, the simplified Bernoulli equation overestimates the actual $T P G_{\text {net }}$ due to the pressure recovery phenomenon [59-65]. The agreement between the estimated value of $T P G_{\max }$ and $T P G_{n e t}$ depends on the magnitude of pressure recovery, which is related to the amount of kinetic energy dissipated downstream from the stenosis. For severe stenoses, significant turbulence production eliminates pressure recovery and $T P G_{\max }$ provides a good aproximation of the pressure gradient. For moderate and mild stenoses, on the other hand, there is not enough turbulence to prevent pressure recovery and the pressure gradient is overestimated [51].

\section{Extended Bernoulli}

Estimations from the simplified Bernoulli equation can be corrected to account for pressure recovery by introducing geometric parameters that indirectly represent the hemodynamic effect of flow expansion. For example, Garcia et al. [61, 66] added an energy loss term to the equation to characterize the geometry of the stenosis and the outflow tract. The resulting pressure gradient can be expressed as follows:

$$
T P G_{\text {net }}=4 v_{\max }^{2}\left(1-\frac{E O A}{A_{A}}\right)^{2}=T P G \max \left(1-\frac{E O A}{A_{A}}\right)^{2} \quad(\mathrm{mmHg})
$$

where $E O A$ is the effective orifice area of the valve, defined as the minimal cross-sectional area of the flow jet (i.e. the cross-sectional area at the vena contracta) and $A_{A}$ denotes the cross-sectional area of the aorta. EOA is determined using the continuity equation $[71,75]$ :

$$
E O A=A_{A} \frac{V T I_{A_{A}}}{V T I_{\max }} \quad\left(\mathrm{cm}^{2}\right)
$$

where $V T I_{A_{A}}$ and $V T I_{\max }$ are the velocity time integral of the systolic velocity curve at the ascending aorta and the vena contracta, respectively.

In vitro evaluation of this approach has demonstrated good agreement between catheter-based measurements and the estimations of $T P G_{\text {net }}$ [66]. In addition, such estimations have been shown to add prognostic value over standard echocardiographic measures of aortic stenosis severity [76].

\subsubsection{D Flow MRI-based Pressure Estimation}

Unlike Doppler echocardiography, 4D Flow MRI yields volumetric velocity data with complete coverage of the cardiovascular regions of interest. This gives access to unique information on flow patterns and therefore allows for a more comprehensive assessment of hemodynamics. With regard to estimation of transstenotic pressure 
gradients, a number of studies have focused on the calculation of the pressure field using 4D Flow MRI velocity data [42, 43, 77-80]. A more recent area of research is the direct quantification of energy losses from the 4D Flow MRI data. Two main approaches have been proposed: the estimation of the energy losses due to turbulence [52] and the assessment of viscous losses in the mean flow field [54].

\section{Pressure Difference Mapping}

Based on the velocity data from 4D Flow MRI acquisitions, pressure differences can be computed between selected points [43], or in the entire volume as 3D or 4D (i.e. time-resolved) pressure maps [42, 77-80]. Typically, the pressure gradients are derived from the 4D Flow MRI-based velocity field using the Navier-Stokes equations. Considering an incompressible, Newtonian fluid, this can be formulated as:

$$
\nabla \mathbf{P}=-\rho \frac{\partial \mathbf{v}}{\partial t}-\rho \mathbf{v} \cdot \nabla \mathbf{v}+\mu \nabla^{2} \mathbf{v}
$$

where $\nabla \mathbf{P}=\left(\frac{\partial P}{\partial x}, \frac{\partial P}{\partial y}, \frac{\partial P}{\partial z}\right)$ is the three-directional pressure gradient field, $\mathbf{v}$ is the $4 \mathrm{D}$ Flow MRI velocity field, $\rho$ is the fluid density and $\mu$ is the dynamic viscosity of the fluid.

The relative pressure differences are then calculated by integrating the pressure gradient field, generally by solving the Pressure Poisson equation (PPE) [42, 78]. A drawback of current pressure mapping using velocities from a conventional 4D Flow MRI acquisition is that it does not account for turbulent effects. Therefore, the applicability of the approach in a stenotic setting is limited [2, 81].

\section{Turbulent Losses}

As previously described, blood flow over a stenosis is characterized by a high velocity jet followed by an area of turbulent flow, with velocity fluctuations and small eddies. Turbulent kinetic energy (TKE) is a direction-independent measure of the energy stored in the fluctuating components, and can be calculated as [68]:

$$
T K E=\frac{1}{2} \rho \sum_{i=1}^{3} \sigma_{i}^{2} \quad\left(\mathrm{Jm}^{-3}\right)
$$

where $\sigma_{i}$ is the intensity of the velocity fluctuations for each principal direction $i=x, y, z$, also referred to as intravoxel velocity standard deviation (IVSD), and $\rho$ is the fluid density. As described in chapter $4, \sigma_{i}$ can be calculated directly from 4D Flow MRI data, by taking advantage of the attenuation in the amplitude of the PC-MRI signal as a result of velocity fluctuations within the voxel [40, 47, 50]. This results in a map of TKE on a voxel-by-voxel basis. TKE corresponds to half the trace of the Reynolds stress tensor [68]. It should be noted that TKE does not provide information about the rates of turbulence production or dissipation. 
Dyverfeldt et al. [52] proposed volumetric integration of TKE (i.e. total TKE ) in the post-stenotic region as an index for predicting $T P G_{\text {net }}$. In a pilot study, the authors reported significantly higher total TKE values in patients with aortic stenosis compared to normals. Furthermore, a very strong correlation $\left(r^{2}=0.91\right)$ was found between peak total TKE in the ascending aorta and the pressure loss index as defined by Garcia et al. [66].

\section{Viscous Losses}

Viscous losses are caused by velocity gradients in the mean velocity field (i.e. laminar viscous losses). These losses increase due to flow features associated with aortic stenosis, such as high speed velocity jets [54], but turbulence effects are not considered.

The viscous dissipation coefficient for each voxel $\left(\phi_{V}\right)$ can be calculated from the first order spatial gradients of the velocities $(v)$ acquired with 4D Flow MRI : $[54,82,83]$ :

$$
\phi_{V}=\frac{1}{2} \sum_{i} \sum_{j}\left[\left(\frac{\partial v_{i}}{\partial x_{j}}+\frac{\partial v_{j}}{\partial x_{i}}\right)-\frac{2}{3}(\nabla \cdot v) \delta_{i j}\right]^{2} \quad\left(\mathrm{~s}^{-2}\right)
$$

where $i$ and $j$ are the principal directions $x, y, z$ and $\delta_{i j}$ is the Kronecker delta [82]. The method provides a map of viscous dissipation values. The rate of energy loss for a volume of interest at a specific time point during the cardiac cycle ( $\left.\dot{E}_{\text {loss viscous }}\right)$ can then be computed as:

$$
\dot{E}_{\text {loss viscous }}=\mu \sum_{i=1}^{N_{\text {voxels }}} \phi_{V} V_{i}
$$

where $\mu$ is the fluid viscosity, $V_{i}$ is the volume of each voxel and $N_{\text {voxels }}$ is the number of voxels within the volume. Barker et al. [54] found a significant increase in viscous losses in patients with aortic stenosis compared to healthy volunteers. Moreover, the values of systolic viscous energy loss in the ascending aorta showed a very strong correlation $\left(r^{2}=0.91\right)$ with estimations of $T P G_{n e t}$ obtained from the extended Bernoulli equation. 


\section{Chapter 6}

\section{Mathematical Modelling}

Mathematical models have become an established method for understanding the underlying mechanisms in cardiovascular physiology. Models enable us to elucidate various phenomena whose explanation from in vivo measurements alone is nontrivial. In addition, they allow to test system-level hypotheses in great detail, and estimate parameters that are difficult or impractical to measure experimentally [1]. Aortic stenosis is an example of a complex, systemic disease. The constriction at the valve causes a rise in LV afterload and intraventricular pressures. Moreover, patients with aortic stenosis may present concomitant systolic hypertension due to increased arterial wall stiffness, further contributing to the elevated LV afterload [62, 84]. In this context, the use of a model that includes: a) the ventricle, b) the aortic valve and c) the arterial system can help to investigate both the isolated and combined effects of the valvular and vascular loads on LV dysfunction.

Models describing hemodynamics in the cardiovascular system vary in complexity, ranging from three-dimensional (3D) models to lumped parameter, zerodimensional (0D) representations. Lumped parameter models generally include several compartments representing the main parts of the system, such as the heart, the valves within it, and different branches of the vascular tree. These models are based on the assumption that the physiological variables of interest (e.g. pressure, flow and volume) are uniformly distributed in space within each compartment. Higherorder models, in contrast, account for continuous spatial variations of these variables. One-dimensional (1D) approaches allow for studying pressure and flow propagation in the vasculature. 3D models are required to obtain complex flow patterns, and are usually applied for investigating detailed flows characteristics at specific regions of the cardiovascular system (e.g. near the heart's valves, within an aneurysm, or at a coarctation) [85].

The choices of dimensionality and type of model depend on the research question and the application for which the model is designed. Paper I involves the study of turbulent flow across a stenotic segment (e.g. aortic valve or aortic coarctation), and therefore we applied a 3D computational fluid dynamics (CFD) simulation approach. In Papers II, III and IV we focus on the study of global hemodynamics and implemented OD, lumped parameter models. These lumped parameter models are mechanistic, in the sense that they obey physical laws and are built based on pre-existing physiological knowledge about the system. 
This section will provide an overview of the methods applied in this thesis for personalizing lumped parameter models of the cardiovascular system. Three main topics are covered: the mathematical formulation of the model, the problem of parameter estimation, and the analysis of model uncertainty.

\subsection{Lumped Parameter Models}

Lumped parameter models are based on an analogy between the blood flow in a compliant vessel and current in an electrical circuit. Blood flow in the cardiovascular system is driven by pressure differences, whereas current in an electrical circuit is driven by differences in voltage. Likewise, frictional losses due to viscous effects, blood inertia, and vessel wall compliance can be modelled using this analogy with a resistor $(R)$, an inductor $(L)$, and a capacitor $(C)$, respectively. By using the hemodynamic-electrical analogy, methods for circuit analysis can be applied to the study of cardiovascular hemodynamics.

Lumped parameter models of the systemic vessels are usually classified into mono-compartment and multi-compartment descriptions. In mono-compartment models, the complete systemic vasculature is represented as a single compartment, characterized by an $R L C$ combination. The first mono-compartment model of the systemic vasculature was the two-element Windkessel model introduced by Otto Frank in 1899 [86]. This representation consists of a combination of a capacitor $C$, which models the elastic properties of the large systemic arteries, and a peripheral resistance determined by the resistor $R$ (Figure 6). This model describes the decay in aortic pressure during diastole using the time constant $\tau=R C$. The twoelement Windkessel model is often used in cardiovascular simulations as a simple approximation of the afterload of the heart, or as a boundary condition for models of higher complexity (e.g. 3D models). This model has subsequently been extended to improve the frequency behavior of the systemic vascular impedance $[87,88]$.

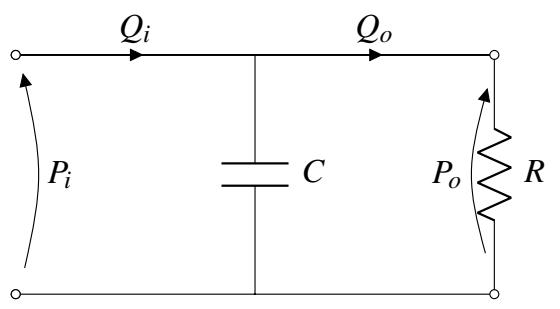

Figure 6: Schematic diagram of the two-element $(R C)$ Windkessel model. $P_{i}$ and $Q_{i}$ are the pressure and flow at the inlet of the vasculature model, respectively. $P_{o}$ and $Q_{o}$ are the pressure and flow at the outlet. 
In multi-compartment models, the systemic vasculature is divided into vessel segments which are combined to obtain an electrical equivalent. Several different models for a vessel segment have been described in the literature [89]. Generally, these models include a set of $R L C$ components. In compliant vessels, like the aorta, all three elements are usually considered. Arterioles and capillaries are relatively rigid, and can be regarded as purely resistive vessels. Therefore, a single resistor is sufficient to characterize their behavior [90]. A typical inverted L-element model of a vessel segment is represented in Figure 7.

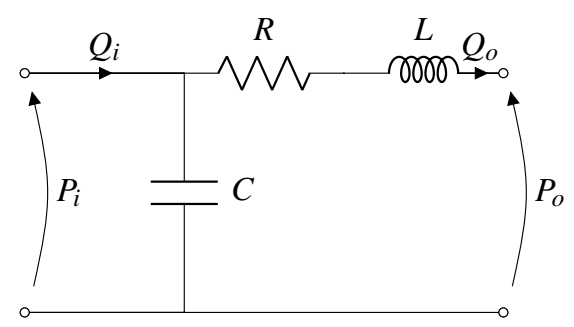

Figure 7: Schematic diagram of an inverted L-element $(R L C)$ vessel segment model. $P_{i}$ and $Q_{i}$ are the pressure and flow at the inlet of the vessel segment model, respectively. $P_{o}$ and $Q_{o}$ are the pressure and flow at the outlet.

The pumping action occurring in each of the heart's chambers is normally represented in lumped parameter models using the time-varying elastance concept [15]. This concept has mainly been used for simulating left ventricular function, although some authors have even applied it to model the left atrium as well [91-93]. The simplest model of the heart valves consists of a diode and a resistor. The diode allows blood to flow through the valve when the pressure gradient across it is positive, while the resistor models the transvalvular pressure gradient.

\subsection{Mathematical Formalism}

The majority of the compartments in lumped parameter models are formulated as systems of ordinary differential equations (ODE). ODEs describe variations in time of the system properties (e.g. pressures, flows, and volumes), and are a wellestablished approach for the study of dynamic data.

The behavior of the system through time is characterized by state variables. These can be interpreted as a set of variables that contain enough information to derive the dynamics of any other variable in the system. The interested reader can find a detailed discussion on the formulation of mathematical models for biological systems in the book by Klipp et al. [94]. 
Mathematically, the state-space model representation can be described as:

$$
\begin{gathered}
\dot{x}=f(x, \theta, u) \\
\hat{y}=g(x, \theta, u) \\
\theta(0)=\theta_{0}
\end{gathered}
$$

where $x$ is the state vector, $\dot{x}$ is the time-derivative of the state vector, $\theta$ is the parameter vector, and $u$ is the input to the system. $x(0)$ denotes the values of the states at time $t=0$. The dependence of $\dot{x}$ on $x, \theta$ and $u$ are determined by the smooth nonlinear function $f . \hat{y}$ are the outputs generated by the model, which correspond to the experimentally measured data. $\hat{y}$ is defined by a smooth nonlinear function $g$. $x, u$, and $y$ are generally time-dependent, but in order to simplify the notation this is not specified, unless required.

Milišić and Quarteroni [89] derived systems of equations to describe lumped parameter models of vessel segments. Each segment gives rise to two ODEs that represent the conservation of mass, and the conservation of energy in that segment. The electrical counterparts to these laws are Kirchoff's current and voltage laws, respectively. The equations for describing the inverted L-element network shown in Figure 7 are defined as follows:

$$
\begin{gathered}
\dot{P}_{i}=\frac{Q_{i}-Q_{o}}{C} \\
\dot{Q}_{o}=\frac{P_{i}-P_{o}-R Q_{i}}{L} \\
P_{i}(0)=0 \\
Q_{o}(0)=0
\end{gathered}
$$

$P_{i}$ and $Q_{o}$ are the state variables of the model. They correspond to the voltage across the capacitor $C$ and the current across the inductor $L . R, L$, and $C$ are model parameters. State-space equations for lumped parameter models with more compartments, including the heart and several segments of the vasculature, can be found in $[95,96]$.

\subsection{Model Personalization}

Personalization involves the calibration of a model's parameters to match experimental observations from a specific subject. Although lumped parameter models have been extensively used for assessing hemodynamics, personalization approaches have been hindered by the limited accessibility of subject-specific measurements [90]. 
Several studies have focused on developing personalized lumped parameter models for their use in critical care. In these models, only a small number of parameters was identified and personalization relied on invasive measurements from porcine in vivo experiments [96-99]. Other authors have proposed personalization approaches based on combining invasive measurements with non-invasive data from echocardiographic LV volumes [100] or PC-MRI flow measurements [101] in patients. The development of personalization approaches that involve exclusively non-invasive measurements has been the subject of recent studies. Keshavarz-Motamed et al. [102] estimated several parameters in a model of the left-sided heart and the systemic circulation using measurements from Doppler echocardiography and twodimensional phase-contrast MRI (2D PC-MRI) to study LV workload in patients with aortic stenosis. More recently, Chabiniok et al. [103] studied mitral valve regurgitation using a lumped parameter model calibrated with measurements extracted from morphological cine MRI images and 2D PC-MRI data.

\subsubsection{Parameter Estimation}

The parameters in the model are typically estimated by solving an inverse problem given the available measurements. The estimation problem involves finding parameter values that are both physiologically feasible, and minimize the deviations between the model output and the experimental measurements. In principle, parameters can be tuned manually to reproduce the experimental data, but this is a time-consuming process that requires extensive physiological knowledge [95]. The most common approach is to use an optimization algorithm to find the parameters that provide the best agreement with the experimental observations. In this thesis, the cost function $V_{N}(\theta)$ is constructed as the sum of squares of the differences between the outputs of the model and the measured data:

$$
V_{N}(\theta)=\sum_{i=1}^{n_{y}} \sum_{j=1}^{N} \frac{\left(y_{i}\left(t_{j}\right)-\hat{y}_{i}\left(t_{j} \mid \theta\right)\right)^{2}}{\sigma_{i}^{2}\left(t_{j}\right)}
$$

where $y(t)$ denotes the measurements, and $\hat{y}(t \mid \theta)$ the simulated outputs. $\mathrm{N}$ represents the number of time points in the measurements, and $n_{y}$ the number of measured signals. $\sigma$ represents the variance of the experimental noise. In this thesis, the variance of the inputs due to measurement noise was not considered, and $\sigma$ was set to one.

The optimization algorithm provides the parameter vector $\hat{\theta}$ that minimizes the cost function, as given by:

$$
\hat{\theta}=\arg _{\theta} \min V_{N}(\theta)
$$

In the optimization routine, the parameter space is spanned and the cost function is evaluated for each combination of parameter values. Usually, upper and lower boundaries are set for each parameter to restrict the search domain. These boundaries are referred to as box-constraints and should be set based on physiologically 
plausible values for each parameter. For a detailed discussion on methods for parameter estimation and model evaluation the reader is referred to the work by Cedersund et al. $[104,105]$.

\subsection{Parameter Uncertainty and Identifiability Analy- sis}

Physiological systems are inherently complex, and often only certain properties can be observed and measured directly. Furthermore, measurements are acquired under specific experimental conditions that may limit their quality or applicability. In the context of medical imaging, the quality of experimental data can be affected by, for instance, the acquisition itself (e.g. spatial and temporal resolution) or the data's signal-to-noise ratio (SNR). The restrictions in the amount and quality of experimental measurements imply that, in general, not all the model parameters can be determined unambiguously. This will result in some parameters being nonidentifiable and others being identifiable within a confidence interval that includes the true value of the parameter. The analysis of how well model parameters are identified given the available data is key in assessing the reliability of the model predictions [104, 106].

\subsubsection{Parameter Confidence Intervals}

A confidence interval $\left[\sigma_{i}^{-}, \sigma_{i}^{+}\right]$at a significance level $\alpha$ for a parameter estimate $\theta_{i}$ indicates that the true value of the parameter $\theta_{i}^{*}$ lies within the confidence interval with probability $\alpha$. Asymptotic confidence intervals can be calculated using the Hessian matrix if the amount of experimental data for the estimation process is large and/or the measurement noise is small. However, this approach is based on the assumption that the observations depend linearly on the model parameters. This is often not the case for mechanistic models and therefore asymptotic confidence intervals are not appropriate [107].

In this work, confidence intervals were determined based on the profile likelihood approach [108]. The profile likelihood method is based on varying the parameter of interest in the same direction, while re-optimizing all the other parameters in the model. By doing this, the parameter space is explored for each parameter in the direction of the lowest increase in the cost function $V(\theta)$. The boundaries of the confidence interval are found when the simulated outputs do not provide an acceptable agreement with the data, as defined by a threshold $\Delta_{\alpha}$. Mathematically, the confidence region is defined as:

$$
\left\{\theta \mid V(\theta)-V(\hat{\theta})<\Delta_{\alpha}\right\}
$$

where $\Delta_{\alpha}$ is the $\alpha$ quantile of the $\chi^{2}(\alpha, d f)$ distribution with $d f$ degrees of freedom, as described in Press et al. [109]. The borders of the confidence region correspond to the confidence intervals [110]. 


\subsubsection{Identifiability Analysis}

The confidence intervals computed using the profile likelihood method enable investigations of the identifiability of each of the model parameters. Identifiable parameters have finite confidence intervals, whereas non-identifiable parameters have confidence boundaries that are infinite in at least one direction. Furthermore, two types of non-identifiability are recognized, namely structural and practical, which are not distinguishable using conventional methods for identifiability analysis [108].
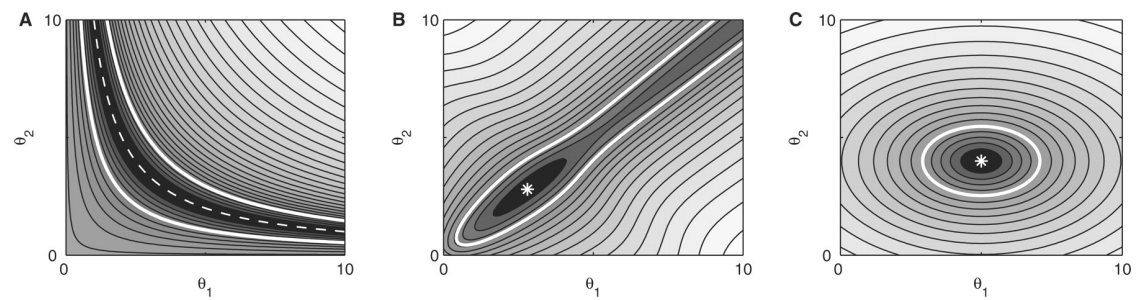

Figure 8: Two-dimensional landscape plots of the cost function $V(\theta)$. Shades from black to white correspond to variations in values of $V(\theta)$, with black and white corresponding to low and high values, respectively. Thick white lines represent likelihood-based confidence regions and white stars the optimal parameter estimates $\hat{\theta}$. Image from Raue et al. [108] (C) 2009 The Authors. Reprinted with permission from Oxford University Press.

Figure 8 shows three different contour plots for the cost function $V(\theta)$ for the parameters $\theta_{1}$ and $\theta_{2}$. Figure $8 \mathrm{~A}$ illustrates the scenario of a structurally nonidentifiable parameter, where the cost function extends infinitely along both directions and the likelihood profile does not have a unique minimum. Structural non-identifiability is a consequence of redundant parametrization of the model and cannot be resolved by increasing the quality of the input measurements (e.g. reducing the noise). This scenario arises from relations between parameters in the model, where the cost function is influenced by this relation but not by each parameter individually. Such a relation can include for instance the sum, the product, or the ratio between two parameters [111].

An example of practical non-identifiability is shown in Figure 8B. In this case, the cost function is infinitely extended in the increasing direction, although there is a minimum in the profile likelihood. For practically non-identifiable parameters, an increase in the amount and quality of the measurement data can in principle lead to identifiability. The information from the profile likelihood analysis can be used to plan new experiments in order to achieve identifiability of practically nonidentifiable parameters and improve confidence intervals [112]. In Figure 8C, the cost function corresponds to an ellipse centered at the optimal parameter estimate, indicating that the parameters are identifiable.

Identifiability analysis is important for assessing the feasibility of the model parameters to be used as potential biomarkers. Biomarkers should be identifiable with a finite confidence interval, indicating that they can be measured given the available data. If the parameter values differ significantly between different subject groups, 
for example between healthy subjects and patients, then the parameter may have a diagnostic value for determining the presence of disease. For instance, Forsgren et al. [113] estimated parameters in a mechanistic pharmacology model of liver function using MRI data and found identifiable parameters that can be used to diagnose liver damage. The use of identifiability analysis in lumped parameter models of the cardiovascular system is relatively recent, as this analysis can be computationally expensive, especially for models with several parameters including a large number of input measurements. As an example, Pironet et al. [99] applied the profile likelihood method to study practical identifiability in a minimal cardiovascular model consisting of seven parameters, personalized with invasive pressure and volume measurements acquired in swine. 


\section{Chapter 7}

\section{Methods and Results}

\subsection{Accuracy of MRI-based Methods for Estimating the Irreversible Pressure Drop}

As discussed in section 5.2, several approaches have been proposed for estimating the irreversible pressure drop across a stenosis [52, 54, 79, 80]. However, despite the increasing number of studies assessing stenosis severity in vivo [52, 114-116], the extent to which the derived non-invasive measurements represent the actual irreversible pressure drop has not been extensively investigated yet. In most studies, evaluation relies on non-invasive measures of either $T P G_{n e t}[52,54]$ or the maximum gradient from the standard Bernoulli approach [79], and comparisons against reference pressure measurements are rarely performed.

In addition, spatial resolution may be a limiting factor in resolving the velocity gradients of the mean flow required to compute some of these parameters. Previous studies have investigated the spatial resolution dependence of viscous losses and pressure gradients obtained using the PPE, and found discrepancies against reference measurements from CFD simulations and particle image velocimetry [41, 83, 117]. However, these studies were limited to a reduced range of flow rates, which are not fully representative of the flow conditions found in patients with varying degrees of stenosis. Therefore, an evaluation of the accuracy of pressure and energy loss measures for a clinically relevant range of stenosis severity is lacking.

In Paper I, "4D Flow MRI-based pressure loss estimation in stenotic flows: Evaluation using numerical simulations", we evaluated the ability of current pressure and energy loss estimations to predict $T P G_{n e t}$, as well as their dependence on spatial resolution, based on simulations of PC-MRI measurements from numerical velocity data obtained using CFD. This approach permits comparison of the estimated parameters against the ground truth values from the CFD simulations, and facilitates investigation for a large number of geometries, flow, and spatial resolution settings.

Three methods for the estimation of pressure gradients were evaluated: the simplified Bernoulli equation, the extended Bernoulli equation and the PPE. Energy losses were quantified using two different parameters: total TKE and viscous energy losses. 


\subsubsection{Numerical Flow Data}

The flow data for simulating the PC-MRI measurements were obtained using CFD simulations of nonpulsatile turbulent flow in a stenotic geometry. The geometry consisted of an idealized circular vessel with a diameter of $14.6 \mathrm{~mm}$ and a cosineshaped stenosis. To account for varying degrees of stenosis, the cross-sectional area of the constriction was reduced to $60 \%, 75 \%$ and $90 \%$ of its initial value. In addition, to replicate the effect of a poststenotic dilation, a geometry with a poststenotic dilation of diameter two times the upstream diameter was considered.

Turbulent flow fluctuations were resolved using Large Eddy Simulations (LES). In LES, the large energy-carrying scales of the turbulent flow are resolved, while the smaller isotropic scales where energy dissipation occurs are modelled [118, 119]. We ensured that the simulations had reached steady state before exporting the data, in order to exclude transient effects from the analysis. The simulations were performed for Reynolds numbers between 500 and 6000, yielding $T P G_{n e t}$ values in the range $0.05-40.4 \mathrm{mmHg}$.

\subsubsection{PC-MRI Simulations}

The high-resolution numerical velocity data from the CFD simulations was used to simulate PC-MRI measurements of the velocity field considering isotropic voxel sizes of $1,1.5$ and $2 \mathrm{~mm}$. For each voxel in the volume, the PC-MRI signal $S_{i}\left(k_{v}\right)$ in each perpendicular direction $i(i=1,2,3)$ was simulated by calculating the Fourier Transform of the velocity distribution within the voxel:

$$
S_{i}\left(k_{v}\right)=\int_{-\infty}^{\infty} s\left(v_{i}\right) e^{-k_{v} v_{i}} \mathrm{~d} v_{i}
$$

where $k_{v}$ denotes the applied motion sensitivity and $s\left(v_{i}\right)$ represents the velocity distribution of the velocity component in the direction $i$. $s\left(v_{i}\right)$ was computed by estimating the probability density function of the velocities within the voxel. To simulate a Gaussian point spread function (PSF), the velocity of each cell $j$ was weighted by a coefficient $w_{j}$ defined by the distance of the cell to the center of the voxel [120]:

$$
w_{j}=\frac{e^{\frac{-d_{j}^{2}}{2 \sigma^{2}}}}{W}
$$

where $d_{j}$ is the distance of the $j$-th cell to the center of the voxel, $W$ the sum of the weights within the voxel and $\sigma$ the variance of the Gaussian function defined from the isotropic spatial resolution $\Delta z$ as $\sigma=\frac{\Delta z}{2.35}$ [21].

The IVSD along the $i$ direction was then computed from the relationship between the magnitudes of the simulated PC-MRI signals acquired at two different motion sensitivities, assuming a Gaussian distribution of velocities within the voxel [40]. Using a non-symmetric flow-encoding scheme, the IVSD is obtained from the simulated measurements at zero motion sensitivity $\left(S_{i}(0)\right)$ and motion sensitivity $k_{v}$ 


\subsection{ACCURACY OF MRI-BASED METHODS FOR ESTIMATING THE IRREVERSIBLE PRESSURE DROP}

$\left(S_{i}\left(k_{v}\right)\right)$, as given by equation 7 . The value of $k_{v}$ was chosen to provide the best accuracy in $\sigma_{z}$ for each of the geometries and flow rates, according to: $k_{v} \cdot \sigma_{z}=1$ [48]. The simulations did not account for noise or saturation effects, and ideal bipolar gradients were considered.

\subsubsection{Results and Interpretation}

Figure 9 shows the relationship between the estimations of pressure and energy loss derived from the PC-MRI measurements and the ground truth value of $T P G_{n e t}$ from the CFD simulations. The estimated gradients from the simplified Bernoulli equation $\left(\triangle P_{\text {simpBernoulli }}\right)$ had a very strong linear relationship with the true $T P G_{\text {net }}$ (Figure 9A) $\left(r^{2}=0.998\right)$. However, as expected, the gradients were overestimated, with larger relative errors (up to $60 \%$ ) for low degrees of stenosis. In general, the relative error was larger for low values of $T P G_{n e t}$ and the geometries with $60 \%$ and $75 \%$ stenosis without poststenotic dilation, which are more susceptible to pressure recovery.

Estimations of $T P G_{\text {net }}$ using the extended Bernoulli equation $\left(\triangle P_{\text {extBernoulli }}\right)$ are shown in Figure 9B. The results showed a very strong linear relationship $\left(r^{2}=0.999\right)$ and were in close agreement with the true $T P G_{n e t}$. Overall, the method was able to compensate for pressure recovery, although errors were still present for low values of $T P G_{n e t}$. Similar to the simplified Bernoulli equation, these errors were associated with geometries with low degrees of stenosis.

Figure 9C shows the estimations of the pressure gradient obtained from the PPE. The estimated gradients $\left(\Delta P_{P P E n e t}\right)$ were close to zero for the entire pressure range. This reflects the inadequacy of this approach for assessing pressure losses, since the mean velocity field used as an input for the pressure calculation does not take turbulent effects into account and therefore cannot reflect pressure losses due to turbulence. On the contrary, the PPE was able to resolve the maximum pressure drop, yielding similar values to those resulting from the simplified Bernoulli equation.

The relationship between the estimations of total TKE and the true $T P G_{n e t}$ is shown in Figure 9D. Total TKE showed a strong linear relationship with $T P G_{\text {net }}$ $\left(r^{2}=0.756\right)$, but the estimations of total TKE were dependent on the flow rate and the geometry of the stenosis. A similar relationship was found between the rate of viscous energy losses $\left(\dot{E}_{\text {loss viscous }}\right)$ and $T P G_{n e t}$, as shown in Figure 9E.

The dependence on spatial resolution of total TKE and $\dot{E}_{\text {loss viscous }}$ is shown in Figure 10. TKE values were relatively independent on spatial resolution (Figure $10 \mathrm{~A}$ ), with an underestimation lower than $11.3 \%$ for all the voxel sizes included in the study. Viscous dissipation losses, on the other hand, were highly dependent on spatial resolution, with increasing underestimation of $\dot{E}_{\text {loss viscous }}$ values as resolution decreased (Figure 10B). The average difference between the $\dot{E}_{\text {loss viscous }}$ values derived from MRI and the ground truth from the CFD simulations was 54\%, 67\% and $75 \%$ for spatial resolutions corresponding to $1,1.5$ and $2 \mathrm{~mm}$, respectively. 

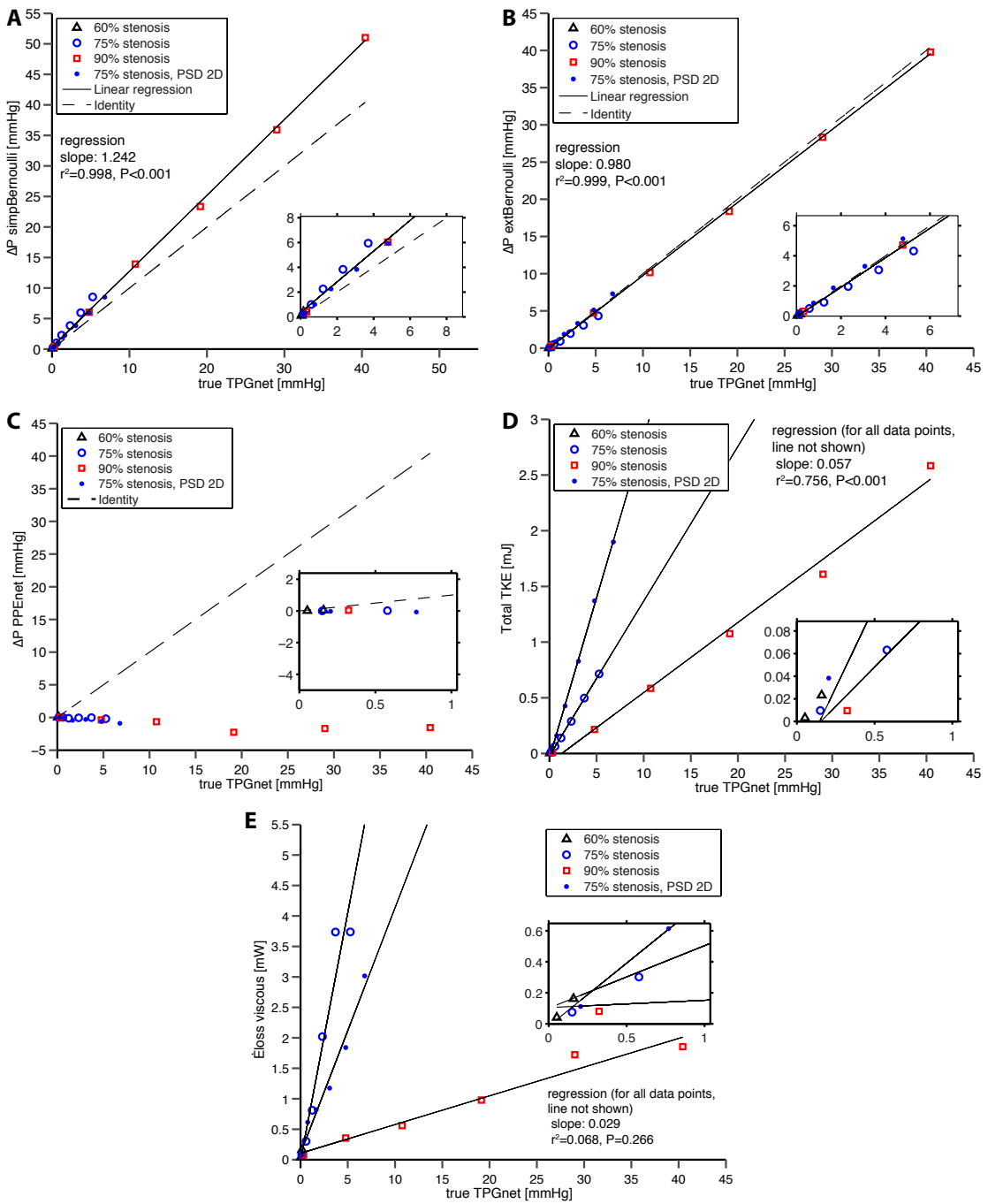

Figure 9: Relationship between the estimated pressure and energy loss measures from the PCMRI simulations and the ground truth value of $T P G_{n e t}$ obtained from the LES solution. The estimations correspond to the pressure gradients from the simplified Bernoulli equation (A), the extended Bernoulli equation (B) and the PPE (C), and energy loss measures including the total TKE (D) and the rate of viscous energy loss $\dot{E}_{\text {loss viscous }}$ (E). The spatial resolution was $1 \mathrm{~mm}$. The solid line represents the linear regression line and the dotted line the identity. For the pressure gradient estimates, the regression line was computed for the entire $T P G_{\text {net }}$ interval. For energy losses, regression analysis was performed for the data corresponding to a specific geometry. The geometry with $60 \%$ area reduction was not included in the analysis. 

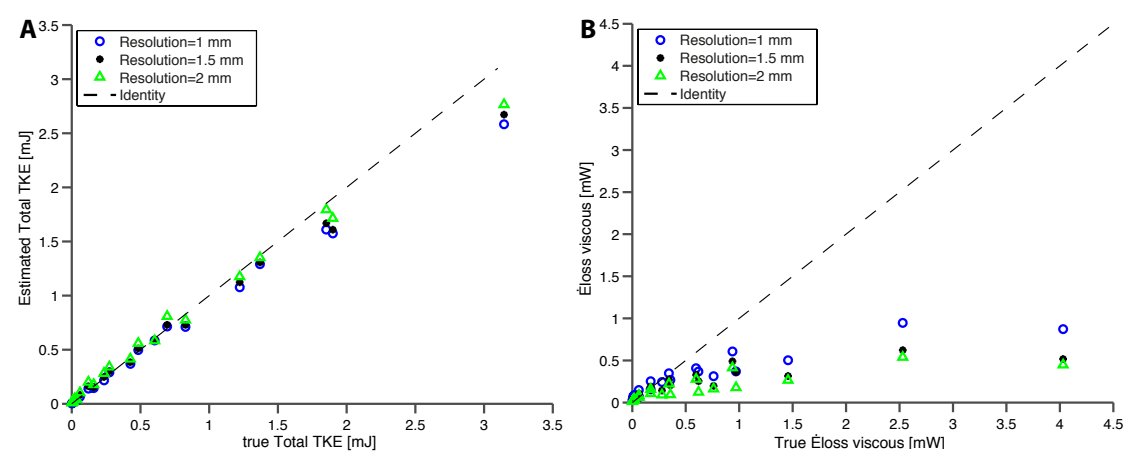

Figure 10: Effect of spatial resolution on the estimations of total TKE (A) and the rate of viscous energy loss $\dot{E}_{\text {loss viscous }}$ (B). The estimations correspond to three different voxel sizes: $1,1.5$ and $2 \mathrm{~mm}$. The dotted line represents the identity.

\subsection{Model Personalization Using 4D Flow MRI}

The clinical usefulness of lumped parameter models is improved when the parameters are tuned to match experimental measurements from a particular subject. However, as discussed in section 6.3, parameter values have traditionally been set based on average population values from literature or invasive measurements from animal studies [96-98, 100], and often only a few parameters can be identified. Some efforts have been made to develop subject specific models using imaging data $[95,102,103,121]$, but personalization typically involves additional invasive measurements [101], hindering eventual incorporation of these modelling approaches into clinical routine.

In Paper II, "Bridging the gap between measurements and modelling: A cardiovascular functional avatar", we propose a framework for constructing a personalized, lumped parameter model of the heart and the systemic circulation using exclusively non-invasive data from routine cardiovascular MR scans and 4D Flow MRI acquisitions.

\subsubsection{The Cardiovascular Model}

The lumped parameter model implemented in this thesis is based on the model introduced by Sun et al. [95]. As shown in Figure 11, the model consists of three main compartments: the pulmonary venous system, the left side of the heart (including the left atrium, the mitral valve, the left ventricle and the aortic valve) and the systemic arterial system. The model of the systemic arterial system is divided into the ascending aorta, the supra-aortic vessels, the descending thoracic aorta, the intercostal arteries, the abdominal aorta and the peripheral tree to the level of the capillary bed. 


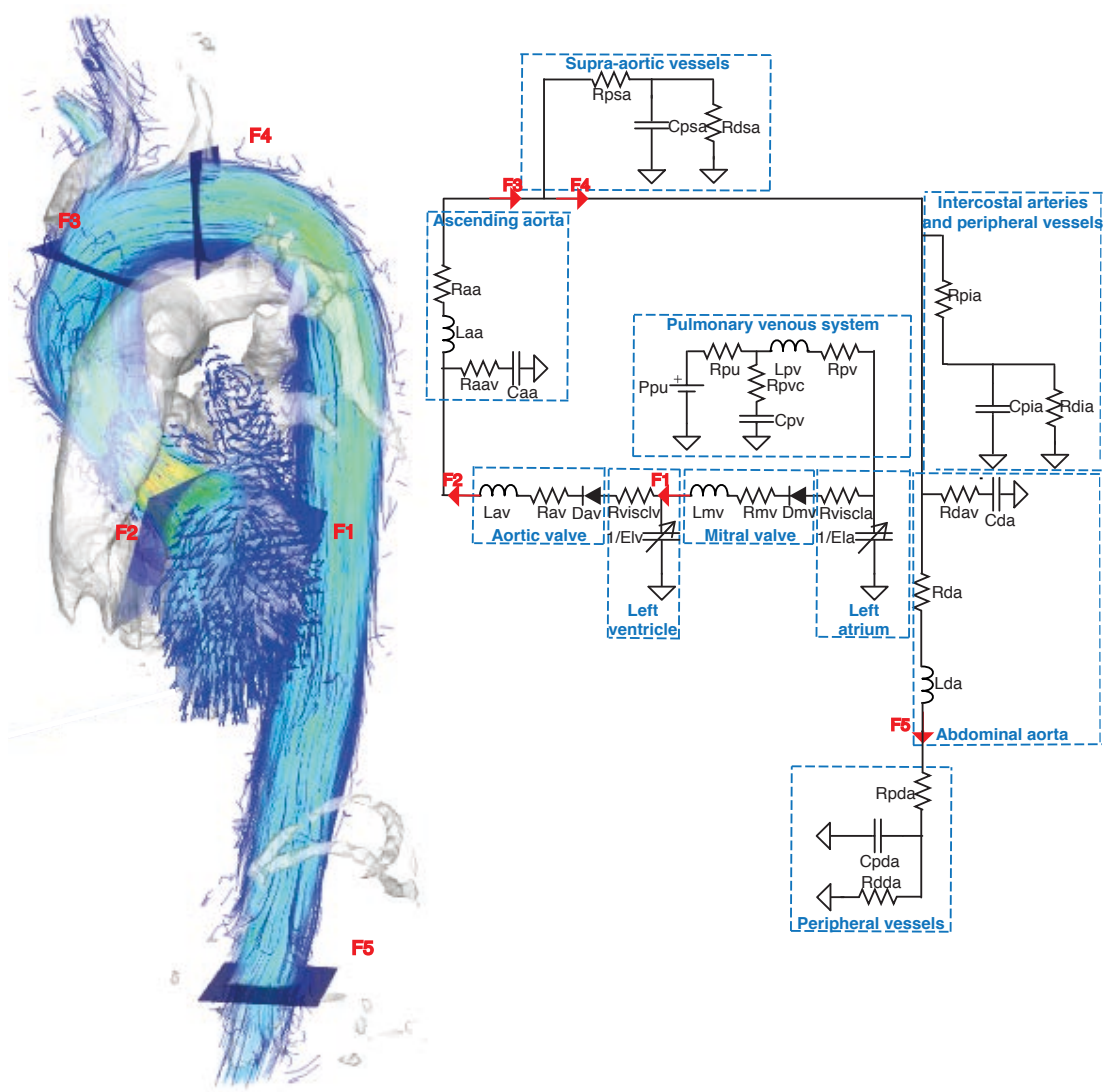

Figure 11: Overview of the approach to personalize the cardiovascular model. Left: Streamline visualization of 4D Flow MRI velocities in the heart and the aorta. The planes indicate the sites where the volumetric flow waveforms $F 1$ to $F 5$ are extracted. Right: Schematic diagram of the lumped parameter model. The red arrows represent the locations of the model-generated flow waveforms involved in the personalization approach. The parameters in the model are tuned so that the model can replicate non-invasive indices of cardiovascular function derived from the measurements and the 4D Flow MRI volumetric flow waveforms. 


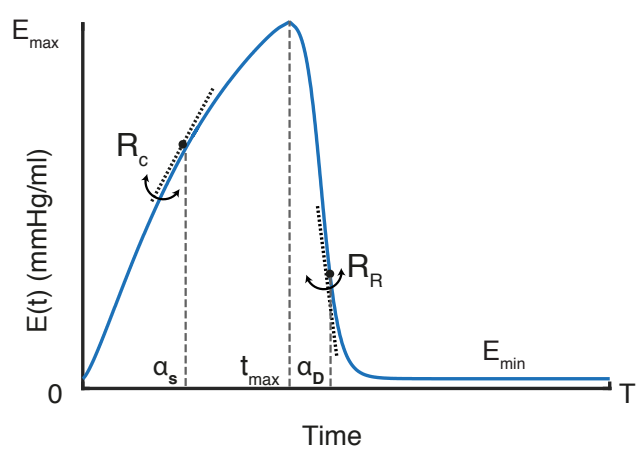

Figure 12: Elastance curve that describes the contractile state of the cardiac chambers. For the ventricle, the elastance gradually increases from the onset of isovolumic contraction $(t=0)$ until the time of end-systole $\left(t=t_{\max }\right)$, when the maximal elastance $E_{\max }$ is achieved. Subsequently, the ventricle relaxes and the elastance decreases until it reaches its minimal value $E_{\min } . R_{C}$, rate of contraction; $R_{R}$, rate of relaxation; $\alpha_{S}$, systolic time constant shape parameter; $\alpha_{D}$, diastolic time constant shape parameter.

The heart chambers were modelled using the time-varying elastance concept [15]. The time course of the elastance $(E(t))$ was approximated by a "double-Hill" function $[122,123]$ :

$E(t)=\alpha\left(E_{\text {max }}-E_{\text {min }}\right)\left(\frac{\left(\frac{t}{\alpha_{S} T}\right)^{R_{C}}}{1+\left(\frac{t}{\alpha_{S} T}\right)^{R_{C}}}\right)\left(\frac{1}{1+\left(\frac{t}{\alpha_{D} T}\right)^{R_{R}}}\right)+E_{\min } \quad(\mathrm{mmHg} / \mathrm{mL})$

where $T$ is the length of the cardiac cycle and $E_{\max }$ and $E_{\min }$ the maximal and minimal elastance of the chamber, respectively. $\alpha$ is a scaling factor that ensures that the maximum value of $E(t)$ is $E_{\max }$. A representative elastance curve is illustrated in Figure 12. The shape of the curve is determined by four dimensionless factors: the rate of chamber contraction $R_{C}$, the rate of chamber relaxation $R_{R}$ and the shape parameters of the systolic and diastolic time constants ( $\alpha_{S}$ and $\alpha_{D}$, respectively). $R_{C}$ controls the slope of the curve during contraction, while $R_{R}$ determines the slope during relaxation. $\alpha_{S}$ and $\alpha_{D}$ control the duration of systole and diastole in relation to $T$.

The aortic valve was model according to the analytical formulation of the transvalvular pressure gradient proposed by Garcia et al. [65], which takes into account the pressure recovery phenomenon. The pressure gradient across the valve was described as:

$$
\Delta P_{a v}(t)=\frac{\rho}{2 E_{L} C o^{2}} Q_{a v}^{2}(t)+L_{a v} \frac{d Q_{a v}(t)}{d t} \quad(\mathrm{mmHg})
$$


where $Q_{a v}(t)$ is the instantaneous flow rate across the valve, $\rho$ is the density of blood, $L_{a v}$ is the inertance of the aortic valve and $E_{L} C o$ is the energy loss coefficient. $E_{L} C o$ is defined as $E_{L} C o=\frac{E O A_{a v} A_{a o}}{A_{a o}-E O A_{a v}}$, where $E O A$ denotes the effective orifice of the valve and $A_{a o}$ is the cross-sectional area of the outflow tract measured at the time of peak systole.

In modelling the aortic valve, pressure recovery was not considered, as its effect on the transmitral pressure is not significant in healthy subjects [124]. Valves were modelled as ideal diodes with two possible states, fully open or closed. Opening of the valves was triggered by a positive pressure gradient.

The model for the vessel segments comprised two resistors ( $R$ and $R_{v}$ ), a capacitor $C$ and an inductor $L$. The resistor $R$ describes viscous losses, $L$ represents mass flow inertia, and the combination of the capacitor $C$ and the resistor $R_{v}$ characterizes the viscoelastic properties of the vessel wall $[95,125]$. The peripheral vascular beds were modelled using three-element Windkessel representations, which include proximal and distal resistances $\left(R_{p}\right.$ and $R_{d}$, respectively) and a capacitor $C$ that characterizes the total compliance.

\subsubsection{Personalization Framework}

The cardiovascular model consists of 50 parameters. Of these parameters, 6 represent the pulmonary venous system, 25 describe the heart chambers and the valves, and 17 characterize the systemic circulation. The duration of the cardiac cycle and the density of blood are also defined as model parameters.

The parameters in the model were personalized using morphological b-SSFP images and 4D Flow MRI data, as well as non-invasive cuff systolic and diastolic pressure measurements in the brachial artery. The b-SSFP images were used for anatomical orientation and segmentation of the left ventricle. The 4D Flow MRI datasets were analyzed to extract volumetric flow waveforms at locations of interest for the model and derive parameters to characterize the morphology and function of the aortic valve.

\section{Subject-specific Input Measurements}

The subject-specific input measurements include the duration of the cardiac cycle, MRI-derived measurements describing the elastance of the left ventricle and the pressure gradient across the aortic valve, brachial arterial systolic and diastolic pressures (SBP and DBP, respectively) and volumetric flow waveforms from five selected sites $F 1$ to $F 5$ (Figure 11) corresponding to the mitral valve $(F 1)$, the aortic valve $(F 2)$, the ascending aorta, upstream from the brachiocephalic trunk $(F 3)$, the aortic arch $(F 4)$ and the abdominal aorta $(F 5)$.

The duration of the cardiac cycle $(T)$ was estimated as the average of the cardiac cycle length during the 4D Flow MRI acquisition. The maximal elastance of the left ventricle $\left(E_{\max }\right)$ was estimated from the brachial arterial SBP. The left ventricular ESV was calculated by manual delineation of the endocardial border in the shortaxis stack at the time of end systole, and an estimation of the unstressed volume 
of the left ventricle $\left(V_{0}\right)$ was obtained using the single-beat method described by Chen et al. [126]. Modelling of the aortic valve, as defined in equation 24, requires estimation of the EOA and $A_{a o}$. The EOA was calculated according to the method described by Garcia et al. [114]. $A_{a o}$ was measured distal to the coronary artery ostia, at the time of peak systole.

Volumetric flow waveforms were extracted at the locations of the heart valves using a retrospective valve tracking approach that includes correction for throughplane motion [35]. Flow waveforms along the aorta were derived from fixed planes. To determine the position of the planes, a 4D PC-MR angiogram was created from the MRI data. The flow through each plane was calculated by manual segmentation of either the valve orifice or the aortic lumen for each time frame. By doing so, time-resolved curves of volumetric flow were obtained at each plane $F 1$ to $F 5$.

\section{Subject-specific Parameter Estimation}

In the personalization approach proposed in this study, 44 parameters were adjusted to be subject-specific using in vivo measurements. The remaining six parameters, which include those describing the pulmonary veins, were set to previously reported values [95].

In brief, the procedure to obtain subject-specific parameters involves two consecutive steps. Initially, a subset of 12 parameters was estimated based on the MRI-measures of cardiac and aortic valve function described previously, as well as global hemodynamic indices such as mean and pulse pressures in the aorta. This subset of parameters includes those describing the time-varying elastance of the left ventricle, the aortic valve and the Windkessel representations of the peripheral vascular beds. In a second step, the remaining 32 parameters were estimated using nonlinear optimization to minimize the deviations between the model-based waveforms and those extracted from the 4D Flow MRI data. The cost function $V_{N}(\theta)$, obtained by particularization of equation 18 , was defined as follows:

$$
V_{N}(\theta)=\sum_{i=1}^{5} \sum_{j=1}^{N}\left(Q_{i}\left(t_{j}\right)-\hat{Q}_{i}\left(t_{j} \mid \theta\right)\right)^{2}
$$

where $\theta$ represents the parameters, $i$ denotes the locations where the flow waveforms $Q_{i}(t)$ were measured, $\hat{Q}_{i}\left(t_{j} \mid \theta\right)$ represents the flow waveforms generated by the model given the parameters $\theta$ and $N$ is the number of time points in each flow waveform. In this study, variability in the inputs due to measurement noise was not considered. The variance term $\sigma_{i}\left(t_{j}\right)$ in equation 18 was set to $\sigma_{i}\left(t_{j}\right)=1$.

\subsubsection{Model Evaluation}

We investigated the capability of the model to simulate pressures and flows in a group of eight healthy volunteers. The subject-specific estimations were evaluated in two different ways. First, we assessed the agreement between the hemodynamic variables generated by the model and the in vivo measurements. Figure 13 shows 


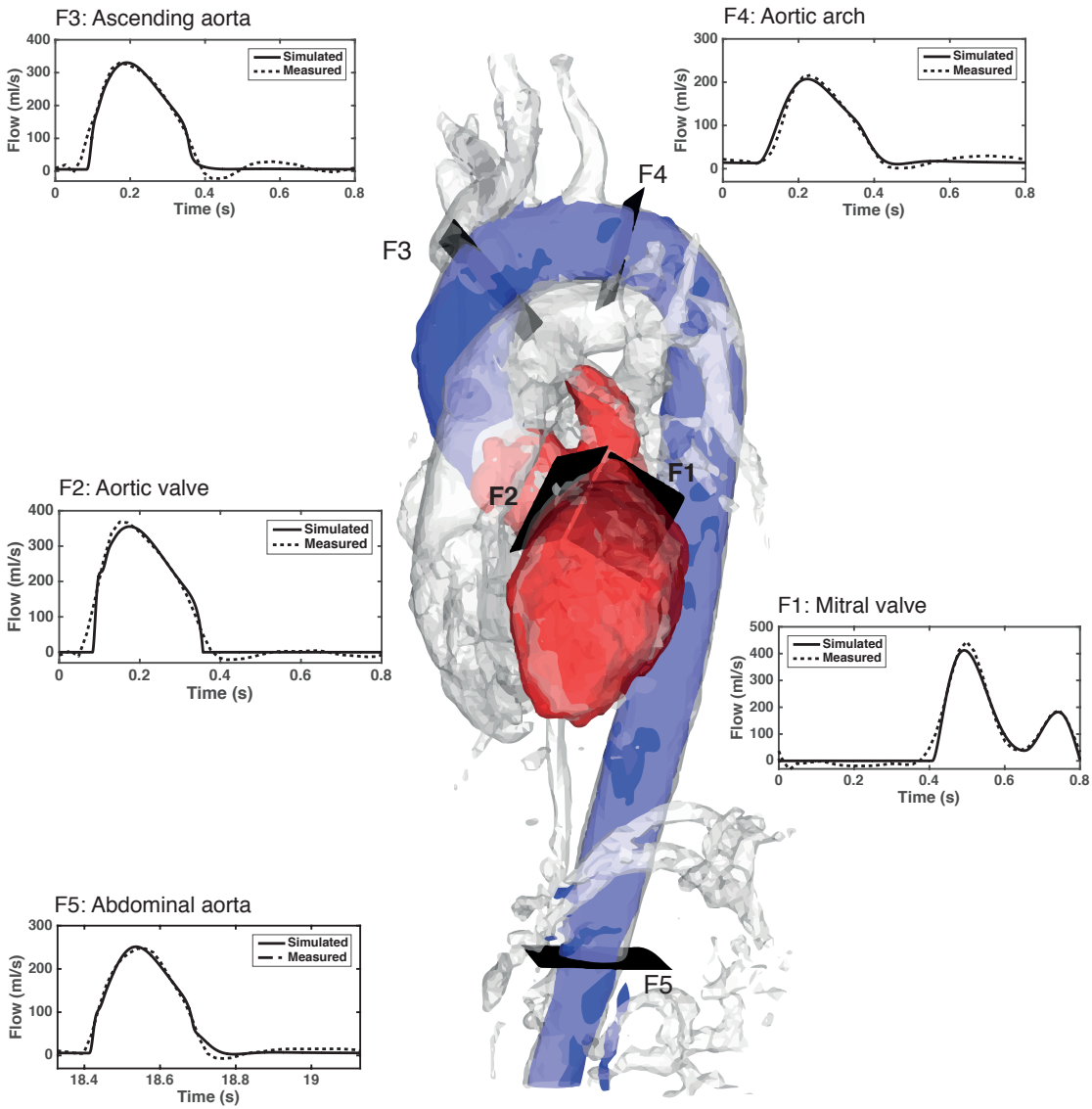

Figure 13: Comparison between the flow waveforms generated by the model (solid lines) and the 4D Flow MRI measurements (dotted lines) at locations $F 1$ to $F 5$ for one representative subject. The subject specific geometry is visualized using an angiogram created from the 4D Flow MRI data, with highlighted regions corresponding to the heart (red) and the aorta (blue). 
A
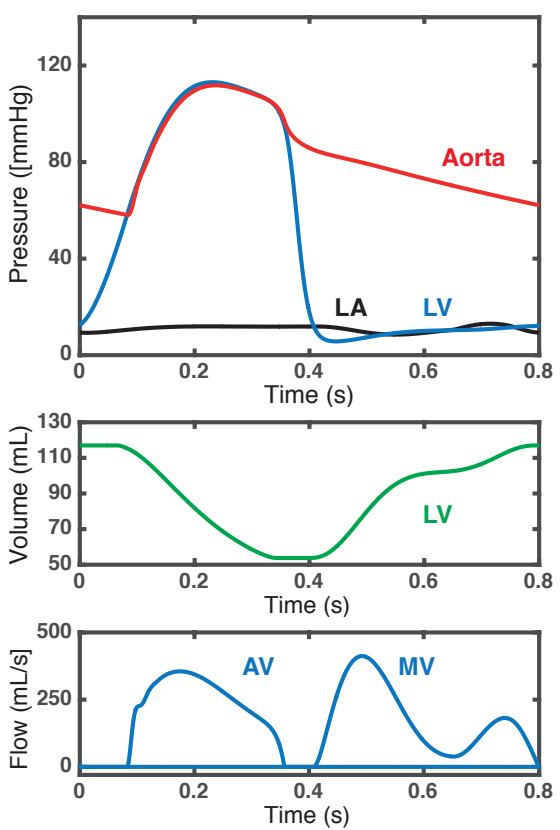

B

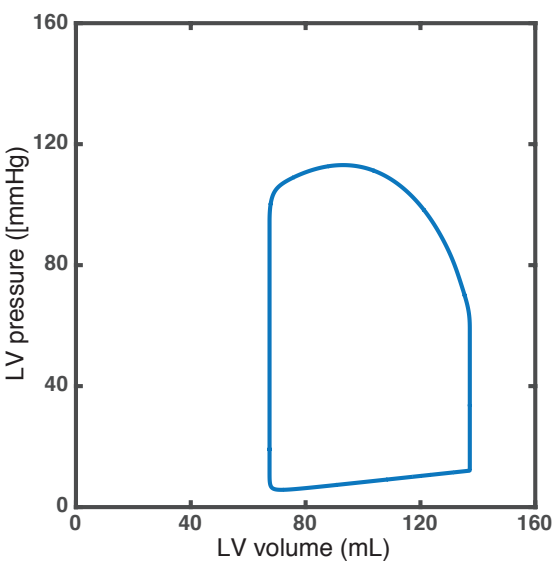

Figure 14: Some of the hemodynamic variables simulated by the model for a representative subject. These include: A) Aortic root pressure, left ventricular (LV) pressure and left atrial (LA) pressure (top panel), left ventricular volume (middle panel), flows across the mitral and the aortic valve (bottom panel). B) Left ventricular pressure-volume loop. AV, aortic valve; LA, left atrium; LV, left ventricle; MV, mitral valve.

a comparison between the flow waveforms generated by the model after personalization and the 4D Flow MRI measurements for one representative subject. The model was able to reproduce the shape of the mitral flow pattern and accurately characterized several flow features, including the amplitude and timing of the early and late filling phases, and the duration of diastasis. Also, the amplitude and the temporal location of the systolic peak in the flow waveforms at the aortic valve, and at the locations along the aorta, were accurately characterized. The values of net flow derived from the measurements and the model-based waveforms at locations $F 1$ to $F 5$ were in good agreement for all subjects in the study.

Figure 14 shows changes in clinically relevant outputs throughout the cardiac cycle for a representative subject. These variables include left ventricular pressures and volumes, pressures in the left atrium and the aorta, and flows across the mitral and the aortic valve (Figure 14A). Across all subjects, the values of SBP generated by the model were in good agreement with the non-invasive cuff measurements, while simulated values of DBP were slightly underestimated. Importantly, the model 
allows to simulate pressure-volume loops (Figure 14B), which cannot be calculated directly from the acquired data and generally require invasive measurements.

The second type of evaluation pertains to the identifiability of the model parameters. Identifiability analysis provides information on the accuracy of the estimated parameters given the experimental measurements. As discussed in section 6.4.2, a parameter is identifiable if it can be determined from the measurements with a finite confidence interval. In this study, identifiability was investigated in one of the study subjects using the profile likelihood method [108]. The results showed that 15 model parameters were identifiable. The parameters describing the time-varying elastance of the left ventricle, the maximal and minimal elastances of the left atrium, and a number of parameters in the circulation (e.g. compliances and resistances) were identifiable. Among the non-identifiable parameters, 11 were practically nonidentifiable and 6 structurally non-identifiable.

\subsection{Model-based Assessment of Cardiac Function Dur- ing Rest and Stress Conditions}

In Paper III, "Non-invasive assessment of systolic and diastolic cardiac function during rest and stress conditions using an integrated image-modelling approach", we applied the modelling approach presented in Paper II to investigate cardiac hemodynamics at rest and under stress conditions. We sought to gain insight into the heart's response to exercise, in terms of left ventricular contraction and relaxation patterns, and derive load-independent parameters quantifying this response that are difficult to measure non-invasively. In particular, we focused on the parameters describing the time-varying elastance of the left ventricle.

Pharmacological stress was induced in nine healthy subjects by infusion of dobutamine. We created two personalized models for each subject in the study, using non-invasive measurements acquired at rest and under dobutamine stress, respectively. As described in Paper II, these measurements included morphological bSSFP images, 4D Flow MRI data and cuff-based pressure measurements. As this study focuses on investigating left ventricular function, we implemented a reduced version of the model presented in Paper II. The model comprises the pulmonary venous system, the left side of the heart, the ascending aorta and a Windkessel model for the systemic vessels. Following model personalization, we performed pairwise comparisons between the subject-specific parameters corresponding to rest and stress conditions using non-parametric Wilcoxon signed-ranked tests.

\subsubsection{Cardiovascular Indices and Flow Measurements}

As expected, dobutamine administration significantly increased heart rate and cardiac output. The individual changes in hemodynamic variables derived from the non-invasive measurements are shown in Figure 15. Systolic blood pressure (SBP) increased significantly, while diastolic blood pressure (DBP) remained unchanged 

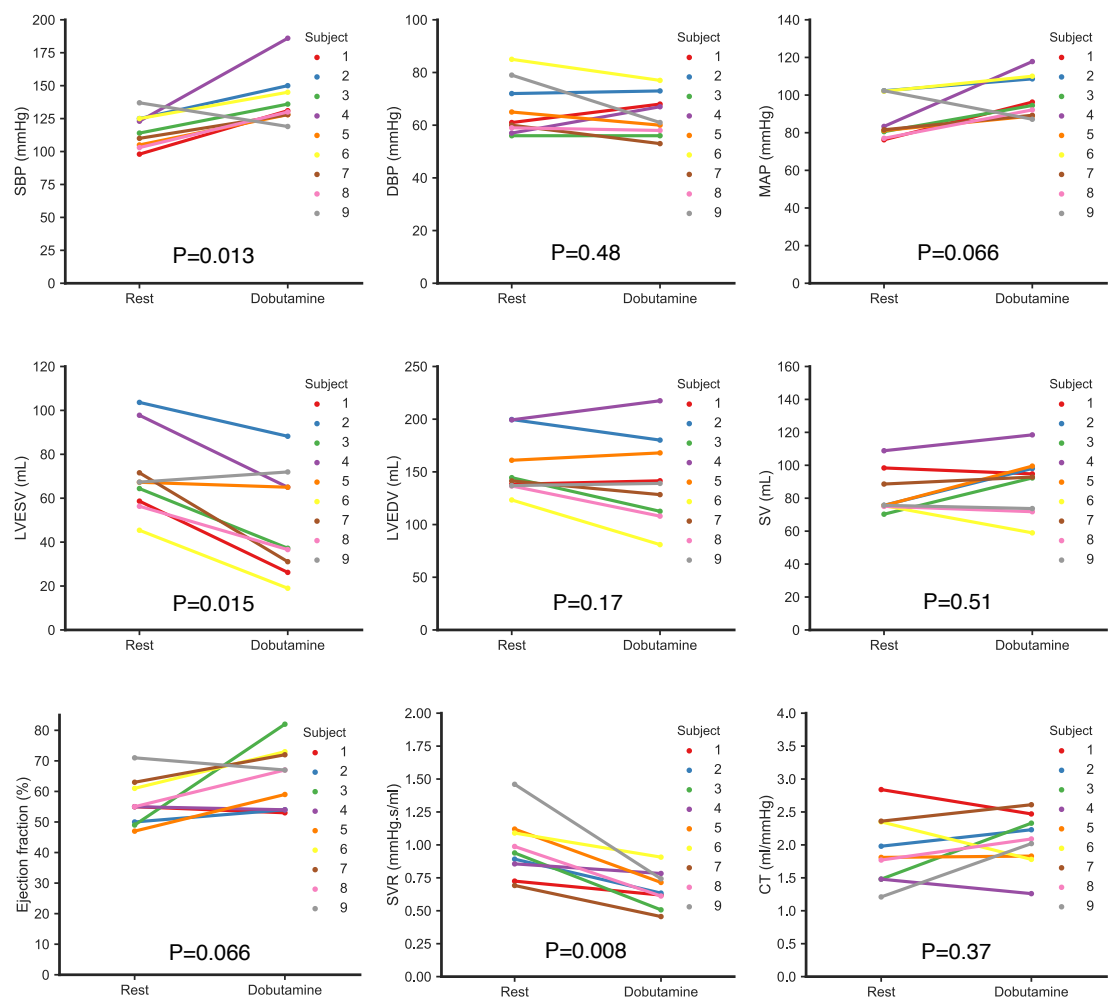

Figure 15: Hemodynamic variables at rest and stress (i.e. after dobutamine infusion) for the nine study subjects. Each subject is represented with a different color. A P value $<0.05$ was considered significant. CT, total systemic arterial compliance; DBP, diastolic blood pressure; LVEDV, left ventricular end-diastolic volume; LVESV, left ventricular end-systolic volume; MAP, mean arterial pressure; SBP, systolic blood pressure; SV, stroke volume. 

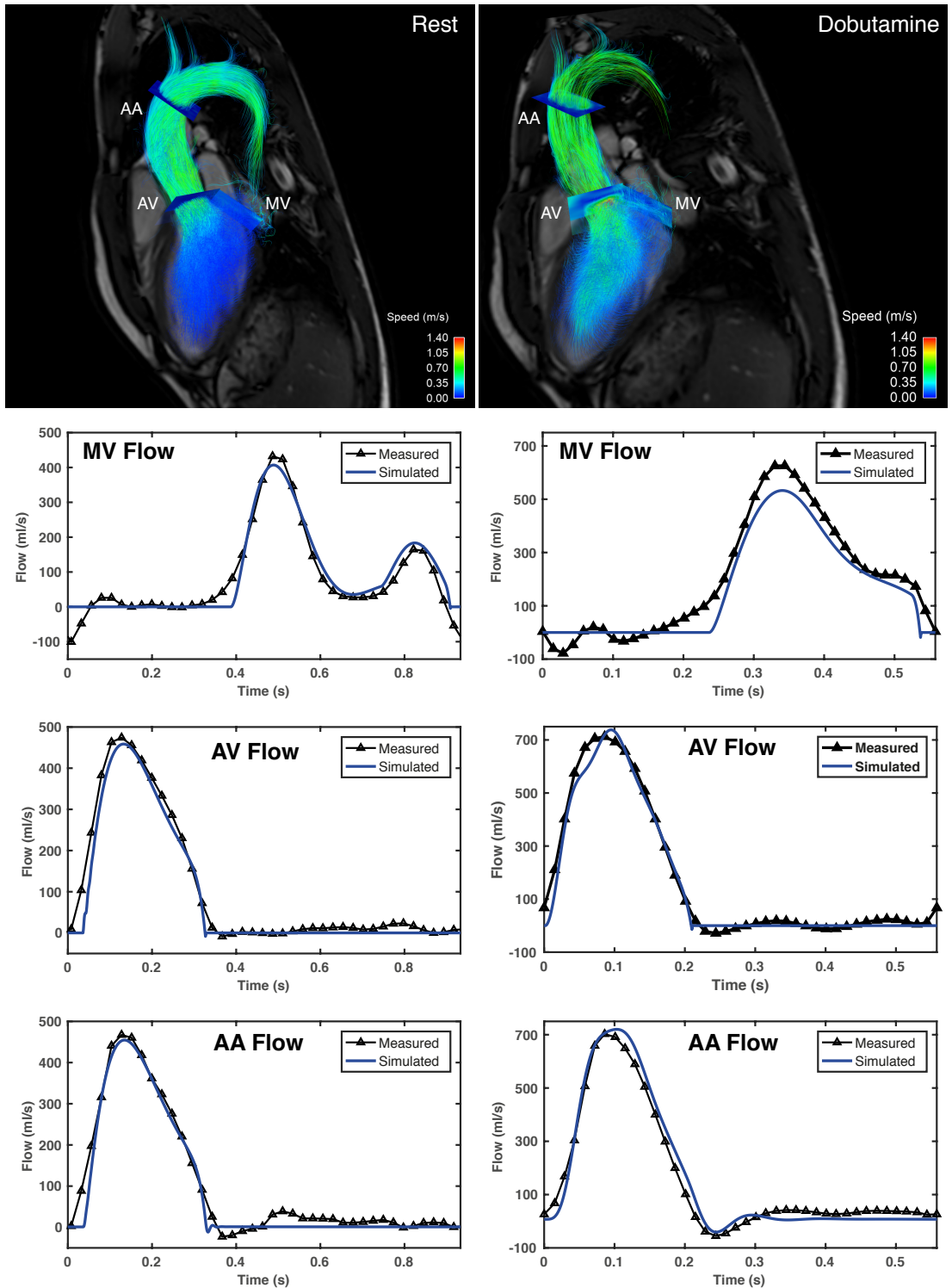

Figure 16: Comparison between model-derived (blue) and 4D Flow MRI (black) volumetric flow waveforms at rest (left) and following the infusion of dobutamine (right) for one representative subject after personalization. The visualizations show flow pathlines covering the complete cardiac cycle, corresponding to the scans performed at rest (left) and following the infusion of dobutamine (right). The planes represent the locations where the flow waveforms were extracted. These locations correspond to the mitral valve (MV), the aortic valve (AV) and the ascending aorta (AA). 

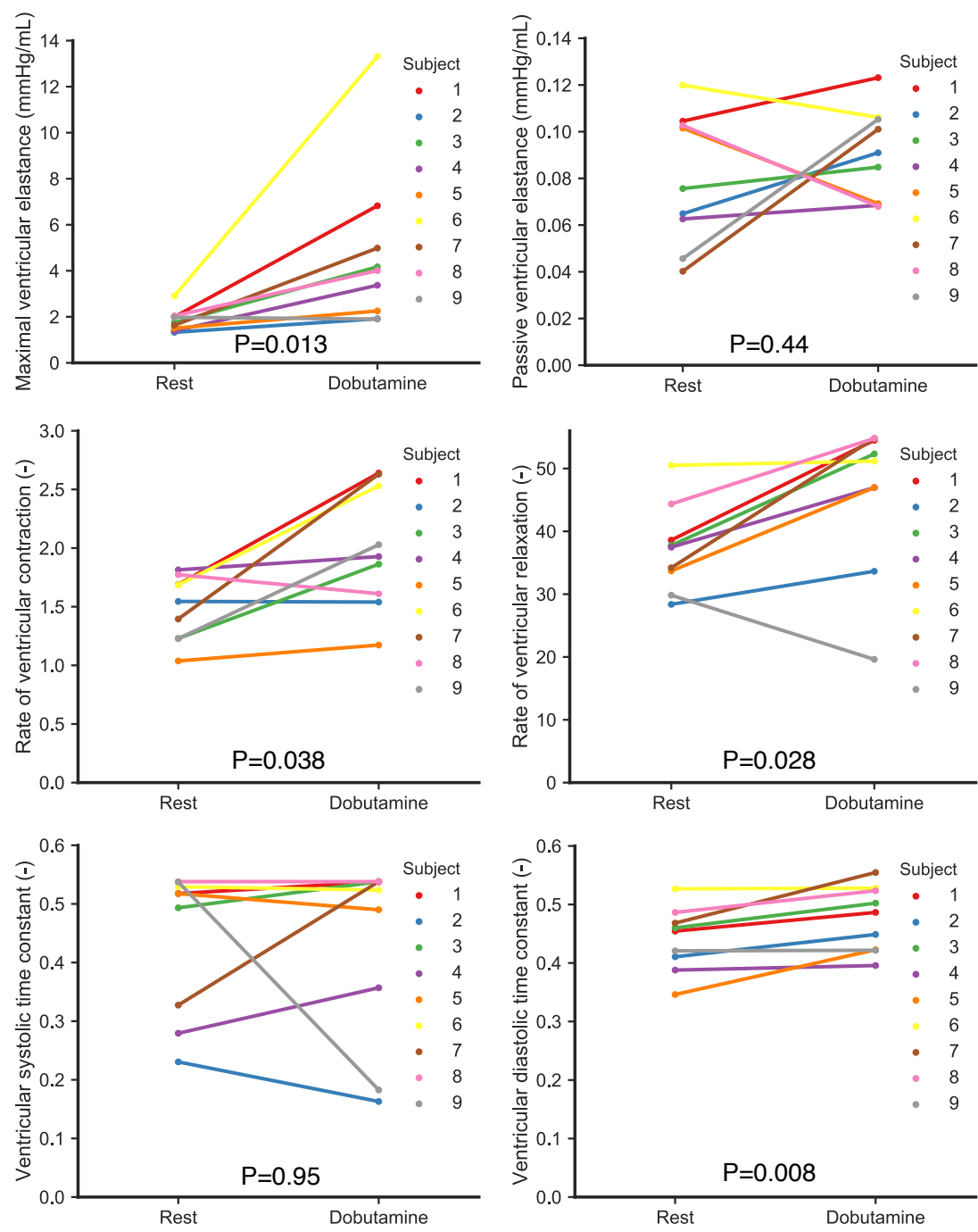

Figure 17: Model-based parameters describing the left ventricular time-varying elastance at rest and following the infusion of dobutamine, for the nine subjects included in the study. The maximal and minimal elastances are related to the amplitude of the time-varying elastance curve, while the contraction and relaxation rates and the time constants determine its shape. Each subject is represented with a different color. A P value $<0.05$ was considered significant 
and mean arterial pressure (MAP) increased non-significantly. We also observed significant increases in left ventricular ESV, while no significant changes were found in EDV, SV or ejection fraction. These results are in agreement with previous studies performing stress tests with MRI and echocardiography [127, 128].

When analyzing the flow waveforms extracted from the 4D Flow MRI data, we found significant increases in peak flows at the aortic valve and the ascending aorta, as well as in the early (E) and late (A) flows at the mitral valve. Furthermore, we observed an increased merging in the $\mathrm{E}$ and $\mathrm{A}$ waves with increasing heart rates, an effect that has been reported in previous studies assessing cardiac function during stress conditions [10, 129]. Figure 16 shows the measured and model-based flow waveforms at rest and following the infusion of dobutamine for a representative subject, where the increase in peak flows and the merging of the E and A waves can be appreciated.

\subsubsection{Time-varying Elastance Parameters}

The changes with dobutamine in model-derived parameters describing the timevarying elastance of the left ventricle are shown in Figure 17. Dobutamine infusion yielded significant differences in the maximal elastance, which is a measure of systolic contractility. The rates of ventricular contraction and relaxation of the ventricle also increased significantly. The increase in left ventricular relaxation rate demonstrates enhanced diastolic function by faster relaxation. These results are in line with previous studies that assessed changes with stress in these parameters invasively [130-132]. Also, as reported by others [10,129], we observed a decrease in the duration of diastole at higher heart rates, as indicated by the significant increase in the diastolic time constant shape factor.

\subsection{Reproducibility of Subject-Specific Model Param- eters}

The results presented in Paper II and Paper III showed that a subgroup of model parameters was identified with a finite confidence interval and behaved appropriately at baseline conditions and when perturbations (i.e. dobutamine infusion) were introduced in the model. In Paper III, we demonstrated the capability of some of these parameters to differentiate between two physiological conditions, corresponding to rest and stress. These parameters may therefore qualify as potential biomarkers. However, if subject-specific model parameters are to be used as biomarkers, they should be robust against errors and variability in the input measurements. The personalization approach presented in Paper II relies on several input measurements that require manual analysis of MRI data, thus being susceptible to acquisition and analysis measurements errors.

In Paper IV, "Reproducibility of 4D Flow MRI-based Personalized Cardiovascular Models; Inter-sequence, Intra-observer, and Inter-observer Variability", we 
investigated the reproducibility of the subject-specific model parameters. We focused on three main sources of variability in the input measurements: intra- and inter- observer variability, and variability related to the MRI acquisition settings (i.e. inter-sequence variability).

Reproducibility was assessed in a group of ten healthy subjects. Each subject underwent two MRI examinations to acquire morphological b-SSFP images and 4D Flow MRI data. Inter-sequence variability was investigated using two different 4D Flow MRI acquisition sequences with different spatial resolution: a spoiled gradient echo (SGRE) sequence with a temporal resolution of $40 \mathrm{~ms}$, and an echo-planar imaging (EPI) sequence with temporal resolution $30 \mathrm{~ms}$.

\subsubsection{Variability in the Input Measurements}

The resulting datasets were analyzed by two different blinded observers. The following measurements were extracted from the datasets: the ESV, the EOA and the volumetric flow waveforms at the mitral valve, the aortic valve and the ascending aorta. To assess the inter-observer variability, one of the observers repeated the analysis of the same set of images. The reproducibility of the input measurements and the subject-specific model parameters was evaluated in terms of the coefficient of variation $(\mathrm{CoV})$ [133]. The resulting values of $\mathrm{CoV}$ for the input measurements derived from the MRI data are listed in Table 1.

\begin{tabular}{cccc}
\hline Input measurement & \multicolumn{3}{c}{$\operatorname{CoV}(\%)$} \\
\hline & Intra-user & Inter-user & Inter-sequence \\
$A_{A o}\left(\mathrm{~cm}^{2}\right)$ & 2.8 & 11.3 & 11.6 \\
ESV $(\mathrm{mL})$ & 3 & 3.8 & 8.6 \\
EOA $\left(\mathrm{cm}^{2}\right)$ & 1.5 & 8.9 & 3.7 \\
\hline
\end{tabular}

Table 1: Repeatability of the input measurements to the model with regard to intra-, interobserver and inter-sequence variability. $A_{A o}$, cross sectional area of the outflow tract; ESV, end-systolic volume; EOA, effective orifice area.

\subsubsection{Intra- and Inter-observer Variability}

For each subject, we created four personalized models, using the input data resulting from the intra-, inter-user and inter-sequence analyses. To construct the models, we used the input data as follows:

- Model 1: Input data from SGRE sequence and first analysis performed by observer 1 .

- Model 2: Input data from SGRE sequence and second analysis performed by observer 1 . 


\begin{tabular}{cccc}
\hline Model parameter & \multicolumn{3}{c}{$\mathrm{CoV}(\%)$} \\
\hline & Intra-observer & Inter-observer & Inter-sequence \\
$E_{\min }(\mathrm{mmHg} / \mathrm{mL})$ & 13.7 & 17.2 & 14.3 \\
$E_{\max }(\mathrm{mmHg} / \mathrm{mL})$ & 4.7 & 5.3 & \\
$R_{C}(-)$ & 6.9 & 9.3 & 10.9 \\
$R_{R}(-)$ & 6.8 & 7.1 & 7.5 \\
$\alpha_{S}(-)$ & 12.9 & 19.4 & 14.9 \\
$\alpha_{D}(-)$ & 2.5 & 3.9 & 3.6 \\
$C_{a a}(\mathrm{~mL} / \mathrm{mmHg})$ & 21.8 & 34.9 & 4.1 \\
\hline
\end{tabular}

Table 2: Repeatability of the subject-specific model parameters with regard to intra-, interobserver and inter-sequence variability. $E_{\min }$, passive LV elastance; $E_{\max }$, maximal LV elastance; $R_{C}$ rate of ventricular contraction; $R_{R}$ rate of ventricular relaxation; $\alpha_{S}$; systolic time constant shape factor; $\alpha_{D}$, diastolic time constant shape factor; $C_{a a}$, compliance of the ascending aorta.

- Model 3: Input data from SGRE sequence and analysis performed by observer 2.

- Model 4: Input data from TFE sequence and analysis performed by observer 2.

It should be noted that, using this approach, the variability in the output parameters does not only account for variability in the input measurements, but also for other sources of uncertainty, such as repeated optimization.

Table 2 shows the values of $\mathrm{CoV}$ related to the subject-specific model parameters. When evaluating intra- and inter-user variability, the parameter with the lowest $\mathrm{CoV}$ values was the diastolic time constant shape factor, $\alpha_{D},(2.5 \%$ and $3.9 \%$, respectively). The parameter with the highest variability was the compliance of the ascending aorta $C_{a a}(21.8 \%$ and $34.9 \%$, respectively). The rest of the parameters showed good to moderate variability, with values of $\mathrm{CoV}$ in the range between $4.7 \%$ and $19.5 \%$. Among the studied parameters, the variability associated with the inter-observer analysis was higher than that from the intra-observer analysis. As the variability in the input measurements was also higher in the inter-observer study, this finding suggests that reproducibility of the output parameters is mainly determined by the analysis steps, rather than the estimation procedure.

\subsubsection{Inter-sequence Variability}

The results in parameter variability from the inter-sequence study (see Table 2) showed similar $\mathrm{CoV}$ to those found in the intra- and inter-observer study. The ventricular diastolic time constant, $\alpha_{D}$, had the lowest coefficient of variation (3.6\%), while $C_{a a}$ had the highest variability. The rest of the parameters had $\mathrm{CoV}$ values in the range $7.5-14.9 \%$. We hypothesize that the high variability in $C_{a a}$ is most 
probably a consequence of the temporal resolution used in this study (40 ms), which may not be sufficient to resolve this parameter [134, 135]. 



\section{Chapter 8}

\section{Discussion and Future Directions}

\subsection{Contributions of this Thesis}

The work presented in this thesis focuses on improving the assessment of cardiovascular function by integrating computational models and images from cardiac MRI acquisitions, including 4D Flow MRI data. The imaging techniques applied here permit access to a variety of anatomical and functional information that has formerly been unavailable or difficult to measure. By integrating this wealth of data with relatively simple models of the cardiovascular system, we were able to provide mechanistic explanations of the underlying physiology and derive hemodynamic parameters that would otherwise require invasive measurements.

Two main approaches to cardiovascular modelling are considered in this thesis. In Paper I, we applied 3D models to study complex flow patterns in a specific region of the cardiovascular system and derived parameters characterizing local hemodynamics. In Paper II, we focused on a system-based approach that considers multiple 0D compartments in the cardiovascular system and the interactions between them. Besides allowing for a comprehensive assessment of global hemodynamics, the use of this type of model facilitates personalization based on imaging data. In this paper, we proposed an approach to estimate subject-specific parameters in a OD model of cardiovascular system using exclusively non-invasive measurements. After personalization, the model can be used to compute clinically relevant indices of cardiovascular function and quantify variables that cannot be assessed directly from the measurements, such as the pressure-volume loop.

The results described in Paper III demonstrate the capability of the approach developed in Paper II to quantify subject-specific changes in cardiovascular function during stress conditions. In Paper IV, we assessed the reliability of the subjectspecific parameters with regard to variability in the imaging data. This is a critical step towards establishing the credibility of the model and including the results it generates into the diagnostic process. 


\subsection{Estimation of The Irreversible Pressure Drop}

The results reported in Paper I reflect the inability of current 4D Flow MRI-based methods to estimate the irreversible pressure drop across a stenosis. As expected, the simplified Bernoulli equation could accurately predict the maximum transstenotic pressure gradient, but the net gradient was overestimated since this approach neglects the occurrence of pressure recovery in the post-stenotic region [136]. This agrees with previous in vivo and in vitro studies, which found an overestimation of the simplified Bernoulli estimations when compared to catheter-based measurements of the irreversible pressure gradient $[60,61,63]$. The inadequacy of the PPE to measure the irreversible pressure gradient was also demonstrated in this study. This is not unexpected, given that the mean velocities from 4D Flow MRI used as input to the equation do not include the effects of turbulence.

The relationship between the measures of energy loss (i.e. total TKE and rate of viscous energy loss) was not linear but instead was dependent on the geometry of the stenosis and the post-stenotic dilation. Furthermore, estimates of viscous dissipation were, in general, underestimated and highly dependent on spatial resolution. Subsequent studies have also reported resolution dependence and underestimation of viscous dissipation values obtained from simulations of PC-MRI measurements based on subject-specific CFD simulations in Fontan patients [137] and particle image velocimetry experiments in a realistic phantom of the aorta [41]. Nevertheless, the use of TKE and viscous dissipation maps may be valuable in visualizing and identifying regions of energy loss in patients with aortic stenosis.

The irreversible pressure drop was best estimated using the extended Bernoulli equation. However, the performance of this method may deteriorate when subject to conditions that deviate from the idealized circular geometries and steady flows employed in this study. Furthermore, this approach heavily relies on accurate estimations of the peak velocity and the EOA. The accuracy of these parameters might be compromised in vivo due to, for instance, the presence of noise.

The results from this study provided additional insights into the limitations of the available methods to resolve irreversible pressure gradients. Since this thesis project was initiated, significant work has been done to estimate pressure and energy losses from 4D Flow MRI data. As discussed in sections 4.4 and 5.2.2, complete characterization of turbulence and the corresponding pressure losses requires estimation of the entire Reynolds stress tensor. The diagonal elements in the stress tensor, which determine TKE, can be obtained using measurements from a conventional 4D Flow MRI acquisition [40]. Although several studies have focused on extending the turbulence mapping approach to measure the full tensor, estimation of the shear stresses has been shown to be challenging due to SNR limitations [50]. However, recently, Haraldsson et al. [51] applied the ICOSA6 motion encoding scheme to quantify the entire Reynolds stress tensor. This encoding scheme improves the dependence on SNR of the shear stresses by using non-orthogonal encoding directions commonly applied in diffusion weighted imaging [138]. Notably, the authors demonstrated that the pressure differences estimated using the PPE were able to reflect pressure losses 
when the Reynolds stresses were included in the calculations.

Recently, we proposed an alternative method to estimate the irreversible pressure drop by quantifying turbulence production using the ICOSA6 acquisition scheme [139]. The method was evaluated by simulation of the PC-MRI signal in a numerical flow phantom. The estimates of the irreversible pressure drop were shown to be independent of the geometry and the post-stenotic dilation, and agreed well with the ground-truth value obtained from the CFD simulations.

Although in vivo feasibility of these emerging approaches is currently under investigation, they show great promise for assessing valvular and vascular pathologies.

\subsection{Creating personalized models using 4D Flow MRI}

In Paper II, we proposed an approach to personalize a lumped parameter of the left heart and the systemic circulation using anatomical and flow data from MRI acquisitions. Model personalization is a challenging task, which depends heavily on the type of data, the analysis techniques applied to obtain model inputs from these data, and the parameter estimation procedure [140].

\subsubsection{Image-Model Fusion}

Previous studies have focused on personalizing lumped parameter models of the cardiovascular system using input data from Doppler ultrasound and 2D PC-MRI measurements [101, 103, 141]. In the context of model personalization, 4D Flow MRI has several advantages compared to these techniques. 4D Flow MRI can provide, in a single acquisition, volumetric velocity data with complete coverage of the cardiovascular regions of interest. Furthermore, it allows for retrospective quantification of the images at any location within the acquired image volume. These distinct features offer unique possibilities for the development of mechanistic approaches, allowing for iterative refinement of the model without the need to perform new data acquisitions. In this iterative process, model predictions guide the analysis of existing data to incorporate additional measurements for further model improvements [142, 143]. As an example, in Paper II, identifiability analysis of the model parameters after personalization revealed that a number of parameters describing the left atrium were practically non-identifiable. This information can be used to design new experiments for resolving these non-identifiabilities. This may be achieved, for instance, by incorporating measurements of the volumetric flow rate through the pulmonary veins into the personalization routine.

\section{Data Extraction}

Typically, personalization requires analysis of the acquired images to extract input data for the model. This is an important step, as inaccuracies in the input measurements will impact the subject-specific parameters and the model predictions $[144,145]$. In the proposed personalization approach, we retrospectively placed 
analysis planes at the regions of interest in the aorta and performed retrospective valve tracking at the heart valves. This may provide more accurate flow waveforms in comparison to 2D PC-MRI measurements for which valve tracking cannot be accomplished [35], thus increasing the accuracy of the subject-specific model outputs. Furthermore, the use of a 4D PC-MR angiogram permitted anatomical orientation, facilitating the placement of analysis planes. Manual analysis of 4D Flow MRI images to generate model inputs is a time consuming process that requires much user interaction, especially when it comes to segmentation of the left ventricle. Therefore, combining the personalized modelling approach with techniques for automatic analysis of 4D Flow MRI data [146] is essential for wider application on larger groups of subjects.

\section{Parameter Estimation}

Parameter estimation was achieved by fitting the model to the subject-specific measurements, minimizing the error between the measurements and the model outputs. Successfully estimated parameters should be able to explain the observations in a satisfactory manner, by providing good agreement with the input data. Importantly, as the estimated parameters are intended to be used as potential biomarkers, it is crucial to consider their identifiability.

In this respect, the choices of model complexity, in terms of the number of adjustable parameters, and the amount of input measurements become increasingly important for successful personalization. Complex models with many parameters generally yield better agreement with the data at the expense of parameter identifiability. Adding to this challenge is the potential risk for overfitting, a phenomenon associated with models that contain too many parameters in relation to the amount of input measurements [147]. Overfitting decreases the reliability of both the estimated parameters and the model predictions [94, 148]. In the proposed personalization approach, the complexity of each model compartment was chosen according to the intended level of detail and the number of available measurements to characterize them. Consequently, in Paper III, the model proposed in Paper II was reduced to focus on the physiology of the left ventricle. In addition, the results from the identifiability analysis performed in Paper II were considered for identifying parameters for model reduction in order to enhance identifiability.

\subsubsection{Model Evaluation}

Evaluation is an essential part in the modelling process and constitutes a critical step towards the application of model-based outputs in clinical routine. In Paper II, we evaluated the proposed modelling approach in two different ways. In the first evaluation, we assessed the capability of the model to reproduce the pressure and flow measurements acquired in vivo. This assessment was performed qualitatively, determining whether the generated flow waveforms captured specific flow features. In addition, the agreement between measured and model-based flow waveforms was assessed in terms of net flow values. It should be noted that this analysis does 
not account for variability (i.e. measurement noise) in the input measurements. Ideally, the evaluation should account for this variability, in order to assess if the agreement between the model output and the measurements is sufficient based on well-established statistical tests (e.g. chi-square test, whiteness test) [104, 149]. However, computing the variability in the measurements derived from the 4D Flow MRI data is far from trivial, as it would depend on a number of factors such as VENC, spatial resolution, and segmentation errors [150,151].

The second evaluation concerns identifiability of the estimated model parameters. Identifiability analysis using the profile likelihood approach revealed that the majority of parameters describing the left ventricle, and a number of parameters characterizing the left atrium and the systemic circulation were identifiable. Nonidentifiable parameters were a consequence of either the structure of the model (i.e. structural non-identifiability) or the measurements used in the estimation (i.e. practical non-identifiability). Nevertheless, full identifiability is not a requirement for model predictions to be valid, as models including non-identifiable parameters can still yield well-determined predictions [149]. A potential limitation of the identifiability analysis performed in this study is that it neglects measurement noise. The presence of noise would most likely decrease the confidence of the subject-specific parameters, although it should not affect the validity of the results in terms of identifiable and non-identifiable parameters.

The analysis performed in Paper II demonstrated the capability of the modelling approach to provide well-determined values of subject-specific parameters that were confined to physiological ranges. In Paper III, we extended this evaluation by applying the model to stress conditions. For this purpose, we investigated the capability of the subject-specific model parameters to describe changes in cardiovascular function due to stress. Analysis of the resulting parameters revealed significant increases in left ventricular contractility and the rates of left ventricular contraction and relaxation, as well as a decrease in the duration of diastole in comparison to the total duration of the cardiac cycle. The agreement of these parameter changes with previously reported effects of stress on cardiovascular function [10, 129-132] constitutes an additional evaluation of the model.

In Paper IV, we further evaluated the robustness of the subject-specific parameter estimates by assessing their dependence on variability in the input measurements. Three sources of variability were considered: intra- and inter-observer analyses, and variability associated with the use of different image sequences. The subject-specific parameters were estimated with good to moderate variability. The use of automatic segmentation techniques would reduce the uncertainty associated to data analysis, improving the clinical applicability of the model predictions.

\subsection{Future Directions}

Despite enhanced imaging capabilities and increasing computing power, the application of cardiovascular models into clinical routine has yet to become a reality. This is partly due to the challenge associated with delivering models that are simple 
enough to be easily translated to the clinic, but capable of addressing clinical needs. In this context, reduced-order, mechanistic models that enable quantitative analysis of complex imaging data are poised to become a powerful tool for the diagnosis and treatment of cardiovascular diseases.

A key contribution of models is the generation of biomarkers. The proposed modelling approach can resolve a number of hemodynamic parameters, including those characterizing left ventricular stiffness and relaxation, that are predictive of alterations in cardiovascular function due to exercise. Hence, these parameters qualify as potential biomarkers and may be used for detection and stratification of diseases. As a next step towards clinical use of these biomarkers, future studies should focus on evaluating their prognostic value beyond currently available measures. This evaluation would be done in a large group of subjects with a spectrum of cardiovascular diseases such as ischemic heart disease, valvular disorders and heart failure. For this purpose, the use of automatic analysis techniques for generating inputs to the model would be of high value. This would allow for creating fully automated subject-specific models that could be applied on large patients cohorts, thus bringing them closer to clinical practice.

A more ambitious goal involves the use of the model as part of a larger, multiscale model for predicting pharmacological effects. Such a model would encompass several temporal and spatial scales, from cell to tissue and organ level (i.e. global hemodynamics). Much progress has been done to simulate the effects of drugs on ion channels [152-154], and at the cellular level by incorporation of these channels into models of cardiac myocytes [155]. The use of a multi-scale model would permit to study the chain of drug effects from intra-cellular to organ level, ultimately contributing to drug development and therapy planning. 


\section{Bibliography}

[1] Pablo Lamata, Ramón Casero, Valentina Carapella, Steve A Niederer, Martin J Bishop, Jürgen E Schneider, Peter Kohl, and Vicente Grau. Images as drivers of progress in cardiac computational modelling. Progress in Biophysics and Molecular Biology, 115(2-3):198-212, 2014.

[2] Petter Dyverfeldt, Malenka Bissell, Alex J Barker, Ann F Bolger, Carl-Johan Carlhäll, Tino Ebbers, Christopher J Francios, Alex Frydrychowicz, Julia Geiger, Daniel Giese, Michael D Hope, Philip J Kilner, Sebastian Kozerke, Saul Myerson, Stefan Neubauer, Oliver Wieben, and Michael Markl. 4D flow cardiovascular magnetic resonance consensus statement. Journal of Cardiovascular Magnetic Resonance, 17(1):1-19, 2015.

[3] John E Hall. Guyton and Hall textbook of medical physiology. Elsevier 2016, 2016.

[4] Arnold M Katz. Physiology of the heart. Philadelphia : Wolters Kluwer Health/Lippincott Williams \& Wilkins Health, 2011.

[5] Donald M Bers. Cardiac excitation-contraction coupling. Nature, 415(6868):198-205, 2002.

[6] Steven M. Pogwizd. Arrhythmogenesis and contractile dysfunction in heart failure - Roles of sodium-calcium exchange, inward rectifier potassium current, and residual beta-adrenergic responsiveness. Circulation Research, 88(11):1159-1167, 2001.

[7] M G Hibberd and B R Jewell. Calcium- and length-dependent force production in rat ventricular muscle. The Journal of Physiology, 329:527-540.1, 1982.

[8] Keith R Walley. Left ventricular function: time-varying elastance and left ventricular aortic coupling. Critical Care, 20(1):270, 2016.

[9] Hiroyuki Suga and Kiichi Sagawa. Mathematical interrelationship between instantaneous ventricular pressure-volume ratio and myocardial force-velocity relation. Annals of Biomedical Engineering, 1(2):160-181, 1972. 
[10] Philip J Kilner, Michael Y Henein, and Derek G Gibson. Our tortuous heart in dynamic mode - an echocardiographic study of mitral flow and movement in exercising subjects. Heart and Vessels, 12(3):103-110, 1997.

[11] Otto Frank. On the dynamics of cardiac muscle. American Heart Journal, 58(2):282-317, 1959.

[12] S W Patterson and E H Starling. On the mechanical factors which determine the output of the ventricles. The Journal of Physiology, 48(5):357-379, 1914.

[13] S A Glantz and R S Kernoff. Muscle stiffness determined from canine left ventricular pressure-volume curves. Circ Res, 37, 1975.

[14] David A Kass. Assessment of Diastolic Dysfunction: Invasive Modalities. Cardiology Clinics, 18(3):571-586, 2000.

[15] Hiroyuki Suga, Kiichi Sagawa, and Artin A Shoukas. Load Independence of the Instantaneous Pressure-Volume Ratio of the Canine Left Ventricle and Effects of Epinephrine and Heart Rate on the Ratio. Circ Res, 32(3):314-322, 1973.

[16] Hiroyuki Suga and Kiichi Sagawa. Instantaneous Pressure-Volume Relationships and Their Ratio in the Excised, Supported Canine Left Ventricle. Circulation Research, 35(1):117-126, 1974.

[17] K M Borow, A Neumann, and J Wynne. Sensitivity of end-systolic pressuredimension and pressure-volume relations to the inotropic state in humans. Circulation, 65(5):988-997, 1982.

[18] K Sagawa, H Suga, A A Shoukas, and K M Bakalar. End-systolic pressure/volume ratio: a new index of ventricular contractility. Am J Cardiol, 40, 1977.

[19] K T Weber, J S Janicki, R C Reeves, L L Hefner, and T J Reeves. Determinants of stroke volume in the isolated canine heart. J Appl Physiol, 37, 1974.

[20] Francis G Spinale. Assessment of Cardiac Function-Basic Principles and Approaches. In Comprehensive Physiology. John Wiley \& Sons, Inc., 2011.

[21] E M Haacke, R W Brown, M R Thompson, and R Venkatesan. Magnetic resonance imaging. Physical principles and sequence design. Wiley-Liss (John Wiley \& Sons), New York, 1999.

[22] Donald W. McRobbie, Elizabeth A. Moore, Martin J. Graves, and Martin R. Prince. MRI from Picture to Proton. Cambridge University Press, 2 edition, 2006. 
[23] P P Dendy and B Heaton. Physics for diagnostic radiology. Series in medical physics and biomedical engineering. Boca Raton, FL : CRC Press, 2011.

[24] Michael Poon, Valentin Fuster, and Zahi Fayad. Cardiac magnetic resonance imaging: a "one-stop-shop" evaluation of myocardial dysfunction. Current Opinion in Cardiology, 17(6), 2002.

[25] Paul R Moran. A flow velocity zeugmatographic interlace for NMR imaging in humans. Magnetic Resonance Imaging, 1(4):197-203, 1982.

[26] Michael Markl, Philip J Kilner, and Tino Ebbers. Comprehensive 4D velocity mapping of the heart and great vessels by cardiovascular magnetic resonance. Journal of Cardiovascular Magnetic Resonance, 13(1):1-22, 2011.

[27] Zoran Stankovic, Bradley D Allen, Julio Garcia, Kelly B Jarvis, and Michael Markl. 4D flow imaging with MRI. Cardiovascular Diagnosis and Therapy, 4(2):173-192, 2014.

[28] Lars Wigström, Tino Ebbers, Anna Fyrenius, Matts Karlsson, Jan Engvall, Bengt Wranne, and Ann F Bolger. Particle trace visualization of intracardiac flow using time-resolved 3D phase contrast MRI. Magnetic Resonance in Medicine, 41(4):793-799, 1999.

[29] Jonatan Eriksson, Carl Johan Carlhäll, Petter Dyverfeldt, Jan Engvall, Ann F Bolger, and Tino Ebbers. Semi-automatic quantification of 4D left ventricular blood flow. J Cardiov Magn Reson, 12(1):1-10, 2010.

[30] Jonatan Eriksson, Ann F Bolger, Tino Ebbers, and Carl-Johan Carlhäll. Fourdimensional blood flow-specific markers of LV dysfunction in dilated cardiomyopathy. European Heart Journal Cardiovascular Imaging, 14(5):417424, 2013.

[31] Philip J Kilner, Guang-Zhong Yang, A John Wilkes, Raad H Mohiaddin, David N Firmin, and Magdi H Yacoub. Asymmetric redirection of flow through the heart. Nature, 404:759, 2000.

[32] Jakub Zajac, Jonatan Eriksson, Petter Dyverfeldt, Ann F Bolger, Tino Ebbers, and Carl-Johan Carlhäll. Turbulent kinetic energy in normal and myopathic left ventricles. Journal of Magnetic Resonance Imaging, 41(4):1021-1029, 2014.

[33] A Fyrenius, L Wigström, T Ebbers, M Karlsson, J Engvall, and A F Bolger. Three dimensional flow in the human left atrium. Heart, 86(4):448-455, 2001.

[34] P M Arvidsson, J Toger, E Heiberg, M Carlsson, and H Arheden. Quantification of left and right atrial kinetic energy using four-dimensional intracardiac magnetic resonance imaging flow measurements. J Appl Physiol, 114, 2013. 
[35] Jos J M Westenberg, Stijntje D Roes, Nina Ajmone Marsan, Nico M J Binnendijk, Joost Doornbos, Jeroen J Bax, Johan H C Reiber, Albert de Roos, and Robert J van der Geest. Mitral Valve and Tricuspid Valve Blood Flow: Accurate Quantification with 3D Velocity-encoded MR Imaging with Retrospective Valve Tracking. Radiology, 249(3):792-800, 2008.

[36] Michael D Hope, Thomas A Hope, Stephen E S Crook, Karen G Ordovas, Thomas H Urbania, Marc T Alley, and Charles B Higgins. 4D Flow CMR in Assessment of Valve-Related Ascending Aortic Disease. JACC: Cardiovascular Imaging, 4(7):781-787, 2011.

[37] Nicholas S Burris and Michael D Hope. 4D Flow MRI Applications for Aortic Disease. Magnetic resonance imaging clinics of North America, 23(1):15-23, 2015.

[38] Hugo G. Bogren, Michael H. Buonocore, and Richard J. Valente. Fourdimensional magnetic resonance velocity mapping of blood flow patterns in the aorta in patients with atherosclerotic coronary artery disease compared to age-matched normal subjects. Journal of Magnetic Resonance Imaging, 19(4):417-427, 2004.

[39] Hojin Ha, Guk Bae Kim, Jihoon Kweon, Sang Joon Lee, Young-Hak Kim, Namkug Kim, and Dong Hyun Yang. The influence of the aortic valve angle on the hemodynamic features of the thoracic aorta. Scientific Reports, 6:32316, 2016.

[40] Petter Dyverfeldt, Andreas Sigfridsson, John-Peder Escobar Kvitting, and Tino Ebbers. Quantification of intravoxel velocity standard deviation and turbulence intensity by generalizing phase-contrast MRI. Magnetic Resonance in Medicine, 56(4):850-858, 2006.

[41] Christian Binter, Utku Gülan, Markus Holzner, and Sebastian Kozerke. On the accuracy of viscous and turbulent loss quantification in stenotic aortic flow using phase-contrast MRI. Magnetic Resonance in Medicine, 76(1):191-196, 2015.

[42] J Michael Tyszka, David H Laidlaw, Joseph W Asa, and Jeffrey M Silverman. Three-dimensional, time-resolved (4D) relative pressure mapping using magnetic resonance imaging. Journal of Magnetic Resonance Imaging, 12(2):321-329, 2000.

[43] Tino Ebbers, Lars Wigström, Ann F Bolger, Jan Engvall, and Matts Karlsson. Estimation of relative cardiovascular pressures using time-resolved three-dimensional phase contrast MRI. Magnetic Resonance in Medicine, 45(5):872-879, 2001. 
[44] A.F. Stalder, M.F. Russe, A. Frydrychowicz, J. Bock, J. Hennig, and M. Markl. Quantitative $2 \mathrm{~d}$ and $3 \mathrm{~d}$ phase contrast mri: Optimized analysis of blood flow and vessel wall parameters. Magnetic Resonance in Medicine, 60(5):12181231, 2008.

[45] Wouter V. Potters, Pim Ooij, Henk Marquering, Ed vanBavel, and Aart J. Nederveen. Volumetric arterial wall shear stress calculation based on cine phase contrast mri. Journal of Magnetic Resonance Imaging, 41(2):505-516, 2015.

[46] Petter Dyverfeldt, Tino Ebbers, and Toste Länne. Pulse wave velocity with 4D flow MRI: Systematic differences and age-related regional vascular stiffness. Magnetic Resonance Imaging, 32(10):1266-1271, 2014.

[47] Petter Dyverfeldt, John-Peder Escobar Kvitting, Andreas Sigfridsson, Jan Engvall, Ann F Bolger, and Tino Ebbers. Assessment of fluctuating velocities in disturbed cardiovascular blood flow: In vivo feasibility of generalized phase-contrast MRI. J Magn Reson Imaging, 28(3):655-663, 2008.

[48] Petter Dyverfeldt, Roland Gårdhagen, Andreas Sigfridsson, Matts Karlsson, and Tino Ebbers. On MRI turbulence quantification. Magnetic Resonance Imaging, 27(7):913-922, 2009.

[49] J H Gao and J O Gore. Turbulent flow effects on NMR imaging: measurement of turbulent intensity. Med Phys, 18:1045 - 51, 1991.

[50] Elkins Christopher, Alley Marcus, Saetran Lars, and Eaton John. Threedimensional magnetic resonance velocimetry measurements of turbulence quantities in complex flow. Experiments in Fluids, 46(2):285-296, 2009.

[51] Henrik Haraldsson, Sarah Kefayati, Sinyeob Ahn, Petter Dyverfeldt, Jonas Lantz, Matts Karlsson, Gerhard Laub, Tino Ebbers, and David Saloner. Assessment of Reynolds stress components and turbulent pressure loss using 4D flow MRI with extended motion encoding. Magnetic Resonance in Medicine, 79(4):1962-1971, 2018.

[52] P Dyverfeldt, M D Hope, E E Tseng, and D Saloner. Magnetic resonance measurement of turbulent kinetic energy for the estimation of irreversible pressure loss in aortic stenosis. JACC. Cardiovasc imaging, 6:64-71, 2013.

[53] Florian von Knobelsdorff-Brenkenhoff, Achudhan Karunaharamoorthy, Ralf Felix Trauzeddel, Alex J Barker, Edyta Blaszczyk, Michael Markl, and Jeanette Schulz-Menger. Evaluation of Aortic Blood Flow and Wall Shear Stress in Aortic Stenosis and Its Association With Left Ventricular Remodeling. Circulation. Cardiovascular imaging, 9(3):e004038-e004038, 2016.

[54] Alex J Barker, Pim van Ooij, Krishna Bandi, Julio Garcia, Mazen Albaghdadi, Patrick McCarthy, Robert O Bonow, James Carr, Jeremy Collins, S Chris 
Malaisrie, and Michael Markl. Viscous energy loss in the presence of abnormal aortic flow. Magnetic Resonance in Medicine, 72(3):620-628, 2014.

[55] Christian Binter, Alexander Gotschy, Simon H Sündermann, Michelle Frank, Felix C Tanner, Thomas F Lüscher, Robert Manka, and Sebastian Kozerke. Turbulent kinetic energy assessed by multipoint 4-dimensional flow magnetic resonance imaging provides additional information relative to echocardiography for the determination of aortic stenosis severity. Circulation: Cardiovascular Imaging, 10(6), 2017.

[56] Florian von Knobelsdorff-Brenkenhoff. Advanced assessment of aortic stenosis reflecting the complex interplay of valve, ventricle, vessel, and flow. Circulation: Cardiovascular Imaging, 10(6), 2017.

[57] Giovanni Cioffi, Pompilio Faggiano, Enrico Vizzardi, Luigi Tarantini, Dana Cramariuc, Eva Gerdts, and Giovanni de Simone. Prognostic effect of inappropriately high left ventricular mass in asymptomatic severe aortic stenosis. Heart, 97(4):301 LP - 307, 2011.

[58] C W Akins, B Travis, and A P Yoganathan. Energy loss for evaluating heart valve performance. Journal of Thoracic and Cardiovascular Surgery, 136(4):820-833, 2008.

[59] Edda Bahlmann, Dana Cramariuc, Eva Gerdts, Christa Gohlke-Baerwolf, Christoph A Nienaber, Erlend Eriksen, Kristian Wachtell, John Chambers, Karl Heinz Kuck, and Simon Ray. Impact of Pressure Recovery on Echocardiographic Assessment of Asymptomatic Aortic Stenosis: A SEAS Substudy. JACC: Cardiovascular Imaging, 3(6):555-562, 2010.

[60] H Baumgartner, T Stefenelli, J Niederberger, H Schima, and G Maurer. "Overestimation" of catheter gradients by Doppler ultrasound in patients with aortic stenosis: a predictable manifestation of pressure recovery. Journal of the American College of Cardiology, 33:1655 - 61, 1999.

[61] Damien Garcia, Jean G Dumesnil, Louis-Gilles Durand, Lyes Kadem, and Philippe Pibarot. Discrepancies between catheter and Doppler estimates of valve effective orifice area can be predicted from the pressure recovery phenomenonpractical implications with regard to quantification of aortic stenosis severity. Journal of the American College of Cardiology, 41(3):435-442, 2003.

[62] Philippe Pibarot and Jean G Dumesnil. Improving Assessment of Aortic Stenosis. Journal of the American College of Cardiology, 60(3):169-180, 2012.

[63] J Niederberger, H Schima, G Maurer, and H Baumgartner. Importance of pressure recovery for the assessment of aortic stenosis by Doppler ultrasound. 
Role of aortic size, aortic valve area, and direction of the stenotic jet in vitro. Circulation, 94:1934 - 40, 1996.

[64] W Voelker, H Reul, T Stelzer, A Schmidt, and K R Karsch. Pressure recovery in aortic stenosis: an in vitro study in a pulsatile flow model. $\mathrm{J} \mathrm{Am} \mathrm{Coll}$ Cardiol, 20:1585 - 93, 1992.

[65] Damien Garcia, Philippe Pibarot, and Louis-Gilles Durand. Analytical modeling of the instantaneous pressure gradient across the aortic valve. Journal of Biomechanics, 38(6):1303-1311, 2005.

[66] D Garcia, P Pibarot, F Sakr, L G Durand, and J G Dumesnil. Assessment of aortic valve stenosis severity: A new index based on the energy loss concept. Circulation, 101(7):765-771, 2000.

[67] C Clark. Energy losses in flow through stenosed valves. Journal of Biomechanics, 12(10):737-746, 1979.

[68] Stephen B Pope. Turbulent Flows. Cambridge University Press, Cambridge, 2000.

[69] P D Stein and H N Sabbah. Measured turbulence and its effect on thrombus formation. Circulation Research, 35(4):608-614, 1974.

[70] S P Sutera. Flow induced trauma to blood cells. Circulation Research, 41(1):28, 1977.

[71] Baumgartner Helmut, Hung Judy, Javier Bermejo, John B Chambers, Thor Edvardsen, Steven Goldstein, Patrizio Lancellotti, Melissa LeFevre, Jr Miller Fletcher, and Catherine M Otto. Recommendations on the echocardiographic assessment of aortic valve stenosis: a focused update from the European Association of Cardiovascular Imaging and the American Society of Echocardiography. European Heart Journal - Cardiovascular Imaging, 18(3):254-275, 2017.

[72] L Hatle, B A Angelsen, and A Tromsdal. Non-invasive assessment of aortic stenosis by Doppler ultrasound. British Heart Journal, 43(3):284-292, 1980.

[73] P S Teirstein, P G Yock, and R L Popp. The accuracy of Doppler ultrasound measurement of pressure gradients across irregular, dual, and tunnellike obstructions to blood flow. Circulation, 72(3):577-584, 1985.

[74] Kieran R O'Brien, Saul G Myerson, Brett R Cowan, Alistair A Young, and Matthew D Robson. Phase contrast ultrashort TE: A more reliable technique for measurement of high-velocity turbulent stenotic jets. Magnetic Resonance in Medicine, 62(3):626-636, 2009. 
[75] H Baumgartner, H Kratzer, and G Helmreich. Determination of aortic valve area by Doppler echocardiography using the continuity equation: a critical evaluation. Cardiology, 77:101-111, 1990.

[76] Edda Bahlmann, Eva Gerdts, Dana Cramariuc, Christa Gohlke-Baerwolf, Christoph Nienaber, Kristian Wachtell, Reinhard Seifert, John Chambers, Karl-Heinz Kuck, and Simon Ray. Prognostic value of energy loss index in asymptomatic aortic stenosis. Circulation, 127, 2013.

[77] T Ebbers, L Wigström, A F Bolger, B Wranne, and M Karlsson. Noninvasive Measurement of Time-Varying Three-Dimensional Relative Pressure Fields Within the Human Heart. Journal of Biomechanical Engineering, 124(3):288293, 2002.

[78] Tino Ebbers and Gunnar Farnebäck. Improving computation of cardiovascular relative pressure fields from velocity MRI. Journal of Magnetic Resonance Imaging, 30(1):54-61, 2009.

[79] Jelena Bock, Alex Frydrychowicz, Ramona Lorenz, Daniel Hirtler, Alex J Barker, Kevin M Johnson, Raoul Arnold, Hans Burkhardt, Juergen Hennig, and Michael Markl. In vivo noninvasive 4D pressure difference mapping in the human aorta: Phantom comparison and application in healthy volunteers and patients. Magnetic Resonance in Medicine, 66(4):1079-1088, 2011.

[80] Hendrik von Tengg-Kobligk, Fabian Rengier, Hendrik von Tengg-Kobligk, Julia Ley-Zaporozhan, Hans-Ulrich Kauczor, Sebastian Ley, Michael Delles, Yoo-Jin Azad, Ruediger Dillmann, Roland Unterhinninghofen, and Joachim Eichhorn. Noninvasive 4D pressure difference mapping derived from 4D flow MRI in patients with repaired aortic coarctation: comparison with young healthy volunteers. International Journal of Cardiovascular Imaging, 31(4):823-830, 2015.

[81] Michael D Hope, S Jarrett Wrenn, and Petter Dyverfeldt. Clinical Applications of Aortic 4D Flow Imaging. Current Cardiovascular Imaging Reports, 6(2):128-139, 2013.

[82] Larry A Glasgow. Transport phenomena: an introduction to advanced topics. Hoboken, N.J. : Wiley, 2010.

[83] Anand K Venkatachari, Sandra S Halliburton, Randolph M Setser, Richard D White, and George P Chatzimavroudis. Noninvasive quantification of fluid mechanical energy losses in the total cavopulmonary connection with magnetic resonance phase velocity mapping. Magnetic Resonance Imaging, 25(1):101-109, 2007.

[84] Martin Briand, Jean G Dumesnil, Lyes Kadem, Antonio G Tongue, Régis Rieu, Damien Garcia, and Philippe Pibarot. Reduced Systemic Arterial Compliance Impacts Significantly on Left Ventricular Afterload and Function in 
Aortic Stenosis. Journal of the American College of Cardiology, 46(2):291 298, 2005.

[85] Frans N van de Vosse and Nikos Stergiopulos. Pulse Wave Propagation in the Arterial Tree. Annual Review of Fluid Mechanics, 43(1):467-499, 2011.

[86] O Frank. Die Grundform des Arteriellen Pulses. Z Biol-Munich, 37:483-526, 1899.

[87] N Westerhof, G Elzinga, and P Sipkema. An artificial arterial system for pumping hearts. Journal of Applied Physiology, 31(5):776-781, 1971.

[88] Nikos Stergiopulos, Berend E Westerhof, and Nico Westerhof. Total arterial inertance as the fourth element of the windkessel model. American Journal of Physiology-Heart and Circulatory Physiology, 276(1):81-88, 1999.

[89] Vuk Milišić and Alfio Quarteroni. Analysis of lumped parameter models for blood flow simulations and their relation with 1D models. ESAIM: Mathematical Modelling and Numerical Analysis, 38(04):613-632, 2004.

[90] Y Shi, P Lawford, and R Hose. Review of zero-D and 1-D models of blood flow in the cardiovascular system. Biomed Eng Online, 10:33, 2011.

[91] G Pennati, M Bellotti, and R Fumero. Mathematical modelling of the human foetal cardiovascular system based on Doppler ultrasound data. Med Eng Phys, 19(4):327-335, 1997.

[92] Mauro Ursino, Andrea Fiorenzi, and Enzo Belardinelli. The role of pressure pulsatility in the carotid baroreflex control: A computer simulation study. Comput Biol Med, 26(4):297-314, 1996.

[93] K Lu, J W Clark, F H Ghorbel, D L Ware, and A Bidani. A human cardiopulmonary system model applied to the analysis of the Valsalva maneuver. American Journal of Physiology - Heart and Circulatory Physiology, 281(6):2661-2679, 2001.

[94] E Klipp. Systems biology : a textbook. Wiley-VCH, 2009.

[95] Y Sun, B J Sjoberg, P Ask, D Loyd, and B Wranne. Mathematical model that characterizes transmitral and pulmonary venous flow velocity patterns. American Journal of Physiology, 268(1 Pt 2):476-89, 1995.

[96] C E Hann, J G Chase, T Desaive, C B Froissart, J Revie, D Stevenson, B Lambermont, A Ghuysen, P Kolh, and G M Shaw. Unique parameter identification for cardiac diagnosis in critical care using minimal data sets. Comput Methods Programs Biomed, 99(1):75-87, 2010. 
[97] C E Hann, J Revie, D Stevenson, S Heldmann, T Desaive, C B Froissart, B Lambermont, A Ghuysen, P Kolh, G M Shaw, and J G Chase. Patient specific identification of the cardiac driver function in a cardiovascular system model. Comput Methods Programs Biomed, 101(2):201-207, 2011.

[98] J Revie, D Stevenson, J G Chase, C E Hann, B Lambermont, A Ghuysen, P Kolh, G M Shaw, S Heldmann, and T Desaive. Validation of subject-specific cardiovascular system models from porcine measurements. Comput Methods Programs Biomed, 2011.

[99] Antoine Pironet, Paul D. Docherty, Pierre C. Dauby, J. Geoffrey Chase, and Thomas Desaive. Practical identifiability analysis of a minimal cardiovascular system model. Computer Methods and Programs in Biomedicine, 2017.

[100] K Sughimoto, F Liang, Y Takahara, K Mogi, K Yamazaki, S Takagi, and $\mathrm{H}$ Liu. Assessment of cardiovascular function by combining clinical data with a computational model of the cardiovascular system. J Thorac Cardiovasc Surg, 145(5):1367-1372, 2013.

[101] Sanjay Pant, Chiara Corsini, Catriona Baker, Tain-Yen Hsia, Giancarlo Pennati, and Irene E Vignon-Clementel. Data assimilation and modelling of patient-specific single-ventricle physiology with and without valve regurgitation. J Biomech, 49(11):2162-2173, 2016.

[102] Zahra Keshavarz-Motamed, Julio Garcia, Emmanuel Gaillard, Romain Capoulade, Florent Le Ven, Guy Cloutier, Lyes Kadem, and Philippe Pibarot. Non-Invasive Determination of Left Ventricular Workload in Patients with Aortic Stenosis Using Magnetic Resonance Imaging and Doppler Echocardiography. PLoS One, 9(1):e86793, 2014.

[103] Radomir Chabiniok, Philippe Moireau, Christoph Kiesewetter, Tarique Hussain, Reza Razavi, and Dominique Chapelle. Assessment of atrioventricular valve regurgitation using biomechanical cardiac modeling. In FIMH 2017 - 9th international conference on Functional Imaging and Modeling of the Heart., volume 10263 of LNCS - Lecture Notes in Computer Science, pages 401-411, Toronto, Canada, 2017. Pop M and Wright G, Springer.

[104] Gunnar Cedersund and Jacob Roll. Systems biology: model based evaluation and comparison of potential explanations for given biological data. FEBS Journal, 276(4):903-922, 2009.

[105] Gunnar Cedersund, Oscar Samuelsson, Gordon Ball, Jesper Tegnér, and David Gomez-Cabrero. Optimization in Biology Parameter Estimation and the Associated Optimization Problem. Uncertainty in Biology, page 177, 2016.

[106] Clemens Kreutz, Andreas Raue, Daniel Kaschek, and Jens Timmer. Profile likelihood in systems biology. FEBS Journal, 280(11):2564-2571, 2013. 
[107] M Joshi, A Seidel-Morgenstern, and A Kremling. Exploiting the bootstrap method for quantifying parameter confidence intervals in dynamical systems. Metabolic Engineering, 8(5):447-455, 2006.

[108] A Raue, C Kreutz, T Maiwald, J Bachmann, M Schilling, U Klingmüller, and J Timmer. Structural and practical identifiability analysis of partially observed dynamical models by exploiting the profile likelihood. Bioinformatics, 25(15):1923-1929, 2009.

[109] William H Press. Numerical recipes in FORTRAN : the art of scientific computing. Cambridge : Cambridge Univ. Press, 1992, 1992.

[110] William Q Meeker and Luis A Escobar. Teaching about Approximate Confidence Regions Based on Maximum Likelihood Estimation. The American Statistician, 49(1):48-53, 1995.

[111] A Raue, C Kreutz, T Maiwald, U Klingmuller, and J Timmer. Addressing parameter identifiability by model-based experimentation. IET SYSTEMS BIOLOGY VO - 5, page 120, 2011.

[112] Bernhard Steiert, Andreas Raue, Jens Timmer, and Clemens Kreutz. Experimental Design for Parameter Estimation of Gene Regulatory Networks. PLoS One, 7(7):e40052, 2012.

[113] Mikael Fredrik Forsgren, Olof Dahlqvist Leinhard, Nils Dahlström, Gunnar Cedersund, and Peter Lundberg. Physiologically Realistic and Validated Mathematical Liver Model Revels Hepatobiliary Transfer Rates for Gd-EOBDTPA Using Human DCE-MRI Data. PLoS One, 9(4):e95700, 2014.

[114] Julio Garcia, Lyes Kadem, Eric Larose, Marie-Annick Clavel, and Philippe Pibarot. Comparison between cardiovascular magnetic resonance and transthoracic doppler echocardiography for the estimation of effective orifice area in aortic stenosis. Journal of Cardiovascular Magnetic Resonance, 13:25, 2011.

[115] Darren P. Lum, Kevin M. Johnson, Russell K. Paul, Aquilla S. Turk, Daniel W. Consigny, Julie R. Grinde, Charles A. Mistretta, and Thomas M. Grist. Transstenotic pressure gradients: Measurement in swine-retrospectively ecggated $3 \mathrm{~d}$ phase-contrast $\mathrm{mr}$ angiography versus endovascular pressure-sensing guidewires. Radiology, 245(3):751-760, 2007.

[116] Julio Garcia, Romain Capoulade, Florent Le Ven, Emmanuel Gaillard, Lyes Kadem, Philippe Pibarot, and Eric Larose. Discrepancies between cardiovascular magnetic resonance and Doppler echocardiography in the measurement of transvalvular gradient in aortic stenosis: the effect of flow vorticity. Journal of Cardiovascular Magnetic Resonance, 15(1):84, 2013. 
[117] A Nasiraei-Moghaddam, G Behrens, N Fatouraee, R Agarwal, E T Choi, and A A Amini. Factors affecting the accuracy of pressure measurements in vascular stenoses from phase-contrast MRI. Magnetic Resonance in Medicine, 52(2):300-309, 2004.

[118] R Gårdhagen, J Lantz, M Karlsson, and F Carlsson. Large Eddy simulation of stenotic flow forwall shear stress estimation - Validation and application. WSEAS Transactions on Biology and Biomedicine, 8(3):86-101, 2011.

[119] J Lantz, M Karlsson, T Ebbers, and J Engvall. Numerical and experimental assessment of turbulent kinetic energy in an aortic coarctation. Journal of Biomechanics, 46(11):1851-1858, 2013.

[120] Umberto Morbiducci, Raffaele Ponzini, Giovanna Rizzo, MarcoEvanghelos Biancolini, Francesco Iannaccone, Diego Gallo, and Alberto Redaelli. Synthetic dataset generation for the analysis and the evaluation of image-based hemodynamics of the human aorta. Medical \& Biological Engineering \& Computing, 50(2):145-154, 2012.

[121] Zahra Keshavarz-Motamed, Elazer R Edelman, Payam K Motamed, Julio Garcia, Nagib Dahdah, and Lyes Kadem. The role of aortic compliance in determination of coarctation severity: Lumped parameter modeling, in vitro study and clinical evaluation. J Biomech, 48(16):4229-4237, 2015.

[122] N Stergiopulos, J J Meister, and N Westerhof. Determinants of stroke volume and systolic and diastolic aortic pressure. American Journal of PhysiologyHeart and Circulatory Physiology, 270(6):2050-2059, 1996.

[123] J P Mynard, M R Davidson, D J Penny, and J J Smolich. A simple, versatile valve model for use in lumped parameter and one-dimensional cardiovascular models. Int J Numer Method Biomed Eng, 28(6-7):626-641, 2012.

[124] Pieter M Vandervoort, Neil L Greenberg, Min Pu, Kimerly A Powell, Delos M Cosgrove, and James D Thomas. Pressure Recovery in Bileaflet Heart Valve Prostheses: Localized High Velocities and Gradients in Central and Side Orifices With Implications for Doppler-Catheter Gradient Relation in Aortic and Mitral Position. Circulation, 92(12):3464-3472, 1995.

[125] Sun Ying. Modeling the dynamic interaction between left ventricle and intraaortic balloon pump. Am J PHysiol-Heart C, 30(4):H1300, 1991.

[126] Chen-Huan Chen, Barry Fetics, Erez Nevo, Carlos E Rochitte, Kuan-Rau Chiou, PhillipYu-An Ding, Miho Kawaguchi, and David A Kass. Noninvasive single-beat determination of left ventricular end-systolic elastance in humans. Journal of the American College of Cardiology, 38(7):2028-2034, 2001.

[127] Katarina Steding-Ehrenborg, Robert Jablonowski, Per M Arvidsson, Marcus Carlsson, Bengt Saltin, and Håkan Arheden. Moderate intensity supine 
exercise causes decreased cardiac volumes and increased outer volume variations: a cardiovascular magnetic resonance study. Journal of Cardiovascular Magnetic Resonance, 15(1):96, 2013.

[128] J D Parker, J S Landzberg, J A Bittl, I Mirsky, and W S Colucci. Effects of beta-adrenergic stimulation with dobutamine on isovolumic relaxation in the normal and failing human left ventricle. Circulation, 84(3):1040-1048, 1991.

[129] Bernard P Paelinck, Hildo J Lamb, Jeroen J Bax, Ernst E van der Wall, and Albert de Roos. MR flow mapping of dobutamine-induced changes in diastolic heart function. Journal of Magnetic Resonance Imaging, 19(2):176-181, 2004.

[130] Joel S Karliner, Martin M Lewinter, Felix Mahler, Robert Engler, and Robert A O'Rourke. Pharmacologic and Hemodynamic Influences on the Rate of Isovolumic Left Ventricular Relaxation in the Normal Conscious Dog. The Journal of Clinical Investigation, 60(3):511-521, 1977.

[131] W C Little, A Rassi Jr., and G L Freeman. Comparison of effects of dobutamine and ouabain on left ventricular contraction and relaxation in closedchest dogs. The Journal of Clinical Investigation, 80(3):613-620, 1987.

[132] Y Myreng and O A Smiseth. Assessment of left ventricular relaxation by Doppler echocardiography. Comparison of isovolumic relaxation time and transmitral flow velocities with time constant of isovolumic relaxation. Circulation, 81(1):260-266, 1990.

[133] Nicole Pauly Hyslop and Warren H White. Estimating Precision Using Duplicate Measurements. Journal of the Air \& Waste Management Association, 59(9):1032-1039, 2009.

[134] Michael Markl, Wolf Wallis, Stefanie Brendecke, Jan Simon, Alex Frydrychowicz, and Andreas Harloff. Estimation of global aortic pulse wave velocity by flow-sensitive 4D MRI. Magnetic Resonance in Medicine, 63(6):1575-1582, 2010.

[135] Fielden Samuel W., Fornwalt Brandon K., Jerosch-Herold Michael, Eisner Robert L., Stillman Arthur E., and Oshinski John N. A new method for the determination of aortic pulse wave velocity using cross-correlation on 2D PCMR velocity data. Journal of Magnetic Resonance Imaging, 27(6):13821387, 2008.

[136] W K Laskey and W G Kussmaul. Pressure recovery in aortic valve stenosis. Circulation, 89(1):116-121, 1994.

[137] Merih Cibis, Kelly Jarvis, Michael Markl, Michael Rose, Cynthia Rigsby, Alex J Barker, and Jolanda J Wentzel. The effect of resolution on viscous dissipation measured with 4D flow MRI in patients with Fontan circulation: 
Evaluation using computational fluid dynamics. Journal of biomechanics, 48(12):2984-2989, 2015.

[138] D G Taylor and M C Bushell. The spatial mapping of translational diffusion coefficients by the NMR imaging technique. Physics In Medicine And Biology, 30(4):345-349, 1985.

[139] Hojin Ha, Jonas Lantz, Magnus Ziegler, Belen Casas, Matts Karlsson, Petter Dyverfeldt, and Tino Ebbers. Estimating the irreversible pressure drop across a stenosis by quantifying turbulence production using 4D Flow MRI. Scientific Reports, 7:46618, 2017.

[140] Radomir Chabiniok, Vicky Y Wang, Myrianthi Hadjicharalambous, Liya Asner, Jack Lee, Maxime Sermesant, Ellen Kuhl, Alistair A Young, Philippe Moireau, Martyn P Nash, Dominique Chapelle, and David A Nordsletten. Multiphysics and multiscale modelling, data-model fusion and integration of organ physiology in the clinic: ventricular cardiac mechanics. Interface Focus, 6(2), 2016.

[141] Patricia Garcia-Canadilla, Fatima Crispi, Monica Cruz-Lemini, Stefania Triunfo, Alfons Nadal, Brenda Valenzuela-Alcaraz, Paula A Rudenick, Eduard Gratacos, and Bart H Bijnens. Patient-specific estimates of vascular and placental properties in growth-restricted fetuses based on a model of the fetal circulation. Placenta, 36(9):981-989, 2015.

[142] Peter Kohl and Denis Noble. Systems biology and the virtual physiological human. Molecular Systems Biology, 5(1), 2009.

[143] T Alexander Quinn and Peter Kohl. Combining wet and dry research: experience with model development for cardiac mechano-electric structure-function studies. Cardiovascular Research, 97(4):601-611, 2013.

[144] Gary R Mirams, Pras Pathmanathan, Richard A Gray, Peter Challenor, and Richard H Clayton. Uncertainty and variability in computational and mathematical models of cardiac physiology. The Journal of Physiology, 594(23):6833-6847, 2016.

[145] Caroline Colijn, Nick Jones, Iain G Johnston, Sophia Yaliraki, and Mauricio Barahona. Toward Precision Healthcare: Context and Mathematical Challenges. Frontiers in Physiology, 8:136, 2017.

[146] Mariana Bustamante, Vikas Gupta, Daniel Forsberg, Carl-Johan Carlhäll, Jan Engvall, and Tino Ebbers. Automated multi-atlas segmentation of cardiac 4D flow MRI. Medical Image Analysis, 49:128-140, 2018.

[147] David A Freedman and David A Freedman. A Note on Screening Regression Equations. The American Statistician, 37(2):152-155, 1983. 
[148] Attila Gábor and Julio R Banga. Robust and efficient parameter estimation in dynamic models of biological systems. BMC Systems Biology, 9:74, 2015.

[149] Gunnar Cedersund. Conclusions via unique predictions obtained despite unidentifiability-new definitions and a general method. FEBS Journal, 279(18):3513-3527, 2012.

[150] Victoria M Stoll, Margaret Loudon, Jonatan Eriksson, Malenka M Bissell, Petter Dyverfeldt, Tino Ebbers, Saul G Myerson, Stefan Neubauer, CarlJohan Carlhäll, and Aaron T Hess. Test-retest variability of left ventricular 4D flow cardiovascular magnetic resonance measurements in healthy subjects. Journal of Cardiovascular Magnetic Resonance, 20(1):15, 2018.

[151] Susanne Schnell, Pegah Entezari, Riti J Mahadewia, S Chris Malaisrie, Patrick M McCarthy, Jeremy D Collins, James Carr, and Michael Markl. Improved semi-automated 4D-Flow MRI analysis in the aorta in patients with congenital aortic valve anomalies vs. tricuspid aortic valves. Journal of computer assisted tomography, 40(1):102-108, 2016.

[152] Jonathan D Moreno, Z Iris Zhu, Pei-Chi Yang, John R Bankston, Mao-Tsuen Jeng, Chaoyi Kang, Lianguo Wang, Jason D Bayer, David J Christini, Natalia A Trayanova, Crystal M Ripplinger, Robert S Kass, and Colleen E Clancy. A Computational Model to Predict the Effects of Class I AntiArrhythmic Drugs on Ventricular Rhythms. Science Translational Medicine, 3(98):98ra83-98ra83, 2011.

[153] Jonathan D Moreno, Pei-Chi Yang, John R Bankston, Eleonora Grandi, Donald M Bers, Robert S Kass, and Colleen E Clancy. Ranolazine for Congenital and Acquired Late I(Na) Linked Arrhythmias: In Silico Pharmacologic Screening. Circulation research, 113(7):e50-e61, 2013.

[154] Colleen E Clancy, Zheng I Zhu, and Yoram Rudy. Pharmacogenetics and anti-arrhythmic drug therapy: a theoretical investigation. American journal of physiology. Heart and circulatory physiology, 292(1):H66-H75, 2007.

[155] Oliver J Britton, Alfonso Bueno-Orovio, Karel Van Ammel, Hua Rong Lu, Rob Towart, David J Gallacher, and Blanca Rodriguez. Experimentally calibrated population of models predicts and explains intersubject variability in cardiac cellular electrophysiology. Proceedings of the National Academy of Sciences of the United States of America, 110(23):E2098-E2105, 2013. 



\section{Papers}

The papers associated with this thesis have been removed for copyright reasons. For more details about these see:

http://urn.kb.se/resolve?urn=urn:nbn:se:liu:diva-151751 